\title{
A Study on the Variability of Kappa $(\kappa)$ in a Borehole: Implications of the Computation Process
}

\author{
by O.-J. Ktenidou, C. Gélis, and L.-F. Bonilla
}

\begin{abstract}
Knowledge of the acceleration spectral shape is crucial to various applications in engineering seismology. Spectral amplitude decays rapidly at high frequencies. Anderson and Hough (1984) introduced the empirical factor $\kappa$ to model this attenuation. This is the first time $\kappa$ is studied in a vertical array consisting of more than two stations. We use 180 earthquakes recorded at a downhole array with five stations in soils and rock to investigate the effect of soil conditions on $\kappa$. Given that $\kappa$ computation processes vary across literature when following the classic AndersonHough method, we investigate its variability with the different assumptions that can be made when applying the method. The estimates of $\kappa_{0}$ range between 0.017 and $0.031 \mathrm{~s}$ at the surface and between 0.004 and $0.024 \mathrm{~s}$ at rock. This variability due to the assumptions made is larger than the error of each estimate and larger than the average difference in values between sediment and rock. For this data set, part of it can be attributed to the type of distance used. Given this variability, $\kappa_{0}$ values across literature may not always be comparable; this may bias the results of applications using $\kappa_{0}$ as an input parameter, such as ground-motion prediction equations. We suggest ways to render the process more homogeneous. We also find that $\kappa$ at rock level is not well approximated by surface records from which we deconvolved the geotechnical transfer function. Finally, we compute $\kappa$ on the vertical component and find a dependence of the vertical-to-horizontal $\kappa$ ratio on site conditions.
\end{abstract}

Online Material: Table of regression parameters and figure showing the regressed lines.

\section{Introduction}

Knowledge of the acceleration spectral shape is crucial to the prediction of ground motion, the generation of stochastic motion, and a number of other applications. At high frequencies, the spectral acceleration amplitude attenuates rapidly. Attenuation can generally be thought of in engineering terms as fractional loss of energy per cycle of oscillation (Lay and Wallace, 1995) or in seismological terms as exponential decrease of amplitude with time or distance (Frankel and Wennerberg, 1987). In either case it consists of two components: one frequency independent, the intrinsic or anelastic attenuation of the material due to friction, and one frequency dependent, the scattering due to heterogeneities in the path. The sum of these can be considered as the inverse of the effective quality factor (Dainty, 1981). The effective attenuation of shear waves within the crust is often modeled using the inverse of the quality factor in exponential relations with distance such as the one given by Futterman (1962):

*Also at Institut des Sciences de la Terre, Université Joseph Fourier, CNRS, BP 53, 38041 Grenoble, Cedex 9, France.

$$
A(R, f)=A_{0} \cdot \exp \left(-\frac{\pi f R}{Q_{\mathrm{ef}} V_{S}}\right),
$$

where $f$ is frequency, $R$ is distance, $Q_{\text {ef }}$ is the effective quality factor, and $V_{S}$ the $S$-wave velocity.

In the 1980s it was observed that this whole-path attenuation was not adequate to explain the attenuation of acceleration spectra at high frequencies, that is, their deviation from the Brune (1970) model above the corner frequency. One of the first to work on this observation was Hanks (1982), who introduced a frequency parameter, $f_{\max }$, above which he observed the high-frequency band limitation of radiated earthquake energy, a phenomenon he called "the crashing spectrum syndrome." Although not altogether excluding source effects as an explanation, he attributed this phenomenon mainly to local site effects. Boore (1983) took into account this highcut frequency filter to model the high-frequency decay in the simulation of synthetic accelerograms through the formula

$$
D(f)=\left[1+\left(\frac{f}{f_{\max }}\right)^{8}\right]^{-\frac{1}{2}} .
$$


Soon after, Papageorgiou and Aki (1983) confirmed this observation but considered its origin tied to the source, suggesting that Hanks' $f_{\max }$ was caused by fault nonelasticity rather than site attenuation, due to the cohesive zone at the crack tip acting as a low-pass filter. In the meantime, Singh et al. (1982) introduced a "site attenuation parameter" to explain the spectral attenuation of $S H$ waves along the Imperial fault. To the exponential path term on the right side of equation (1), they added an exponential site term: $\exp \left(-\pi f t^{*}\right)$. Cormier (1982) proposed a similar formulation. The $t^{*}$ factor was the predecessor of what came to be one of the best known and most frequently used factors to model high-frequency spectral attenuation: the "spectral decay factor" introduced by Anderson and Hough (1984) and denoted as $\kappa$ (kappa; a term also adopted by Singh et al., 1989). This factor stemmed from the observation that in log-linear space the decay of the acceleration spectrum can be considered as linear for frequencies higher than a specific frequency $f_{e}$ :

$$
A(f)=A_{0} \cdot \exp (-\pi \kappa f), \quad f>f_{e} .
$$

This formula holds true under the assumption that the effective quality factor (i.e., the overall attenuation) is frequency independent; $\kappa$ can be simply related to the slope $\lambda$ of the acceleration spectrum $(a)$ over a certain range of frequencies $(\Delta f)$ starting at $f_{e}$, when spectral values are plotted in natural log scale and frequency values are plotted linearly (see fig. 2 of Anderson and Hough, 1984):

$$
\kappa=-\lambda / \pi, \quad \text { where } \quad \lambda=\Delta(\ln a) / \Delta f .
$$

Anderson and Hough (1984) also observed a correlation between observed values of $\kappa$ and the distance of the source from the station where the record was obtained. They suggested a linear relation where the intercept of the $\kappa$ trend with distance (denoted $\kappa_{0}$ ) corresponds to the attenuation $S$ waves encounter when traveling vertically through the shallow geology, and the slope of the trend corresponds to the incremental attenuation due to predominantly horizontal $S$-wave propagation through the crust. If the trend is denoted by $\kappa_{R}$ then the relation can be written as follows, in units of time:

$$
\kappa=\kappa_{0}+\kappa_{R} \cdot R(s) .
$$

This relation derived from the observation that for sites with different local soil conditions, the intercept differed systematically according to those conditions but the distance dependence was not correlated to them and thus was considered similar and attributed to regional effects. Anderson and Hough (1984) and Hough et al. (1988) admit that there is no reason to assume a linear dependence on distance other than the simplicity of the formulation. Anderson (1991) suggested an alternative nonlinear formulation for the distance dependence of $\kappa$, where the dependence can be given by any smooth function of distance. In more recent years, $\kappa$ has become a subject of debate, and there have been studies to estimate its dependence on various factors and to decipher its origins. Gentili and Franceschina (2011) use an inversion scheme to determine $\kappa$ dependence with distance and find it to be concave, which they then model with a piecewise linear function with two different slopes. Fernández et al. (2010), on the other hand, derive a convex dependence with distance, whereas Kilb et al. (2012) find no significant correlation with distance, albeit for distances $<40 \mathrm{~km}$.

Tsai and Cheng (2000) propose an alternative model where $\kappa$ depends primarily on the source, secondarily on the site, and only slightly on distance. Purvance and Anderson (2003) suggest that $\kappa$ depends strongly on source effects (magnitude and focal mechanism) and attribute the lack of distance dependence to frequency-dependent $Q$. Following up on the Papageorgiou and Aki (1983) model, Halldorsson and Papageorgiou (2005) again suggest that $\kappa$ is source related. Boore (2003), who refers to $\kappa_{0}$ as path-independent diminution, considers it may be due either to source or site effects, whereas Cotton et al. (2006) consider it a site parameter. After revisiting the ANZA network where the notion of $\kappa$ started, and comparing four definitions of $\kappa$, Kilb et al. (2012) find it to be site dependent with scatter that is source dependent. Because of its empirical nature, the debate as to the origins and physical meaning of $\kappa$ is still ongoing, as is the debate for its correlation to more rigorously defined parameters such as $Q$ (see the comprehensive paper of Campbell, 2009, for a fuller background review).

Despite the ongoing debate regarding its origins and though it lacks a rigorous theoretical basis, $\kappa$ is often used in a variety of applications today, almost 30 years after its definition. It is used in the computation of site amplification factors when using the quarter-wavelength method of Joyner et al. (1981) and Boore and Joyner (1997). It constitutes an important input parameter in the prediction of ground motion and thus in the creation and calibration of ground-motion prediction equations (GMPEs), particularly for central and eastern North America (Toro et al., 1997; Campbell, 2003; and Atkinson and Boore, 2006). Also, because these are usually created for hard rock sites, $\kappa$ is also used when adjusting such models for softer site conditions. This may be done through the host-to-target method used, for example, by Cotton et al. (2006), Douglas et al. (2006), and Van Houtte et al. (2011). $\kappa$ is also necessary to constrain the attenuation, peak ground acceleration (PGA), and spectral shape of synthetic accelerograms generated with stochastic methods in ground-motion simulation studies (e.g., Boore, 2003). It is also computed on an individual event basis (without the regression that gives $\kappa_{0}$ ) to correct records for site effects before these can be used in studying the spectral characteristics of the seismic source (e.g., Margaris and Hatzidimitriou, 2002; Oth et al., 2010; Lancieri et al., 2012). Finally, $\kappa_{0}$ may be used as an indicator of local site conditions by comparing values at specific sites with typical values, especially given empirical correlations of $\kappa_{0}$ with $V_{S 30}$ such as those introduced by Silva et al. (1998). Thus, it is also studied in the context of site characterization and site effect studies 
(e.g., Drouet et al., 2010). Recently it has been correlated also to other engineering parameters such as the Arias intensity and PGA (Mena et al., 2010).

Our motivation in this study lies in this large field of applications for which $\kappa$ is an important input parameter, in conjunction with the observation that different researchers may implement the Anderson and Hough (1984) method in different ways. Even if equation (5) is used, several assumptions can be made in the process of computing $\kappa$, and there is no general agreement in how these are dealt with across literature. Given the field of application of $\kappa$, we consider that this variability in the method of computation may affect the accuracy and variability of $\kappa$ as an input parameter for the aforementioned uses. For this reason, we use an available data set from a downhole array to make a sensitivity study and examine different factors that may affect $\kappa$. Also, given the availability of downhole records, we also examine the effect of soil properties $\left(V_{S}\right)$ at different stations. More than provide a single answer as to the value of $\kappa$ in the area under study, this work aims to draw attention to the possible variability of the estimates based on the decisions made in the process.

In the present work, we assume the model of equation (5), where $\kappa_{0}$ is a site effect, accepting the underlying assumption of frequency-independent $Q$. This is still one of the most widely used methods despite the existence of alternative approaches, for example, use of displacement spectra (Biasi and Smith, 2001), fitting of observed response spectra (Silva et al., 1998), and full inversions for source, path, and site parameters (Drouet et al., 2010; Oth et al., 2011). We assume a linear dependence of $\kappa$ with distance, which we justify a priori by its simplicity and a posteriori by the high coefficients of correlation computed for the lines. Moreover, we note that in this work the aim is not to correlate $\kappa$ with $Q$ (as done, e.g., in Hough and Anderson, 1988; Hough et al., 1988) but rather to focus on the computation of $\kappa$, per se, as defined by Anderson and Hough (1984) via equation (5) and implemented even recently by Douglas et al. (2010) and Van Houtte et al. (2011).

\section{Study Area and Data Set Used}

The area studied is the city of Aegion, located in the southwestern part of the Gulf of Corinth, Greece, one of the most active seismic areas in Europe. Significant earthquakes have struck the city in the past, the strongest recent one being that of 15 June $1995\left(M_{\mathrm{S}}=6.2\right)$, which caused severe damage to buildings and loss of lives. The PGA recorded was nearly $0.5 \mathrm{~g}$, one of the strongest ever recorded in Greece. The city is crossed by a fault the escarpment of which divides it into two levels, and the site is also marked by the edge of a sedimentary basin extending to the north (Fig. 1). Our data set comes from the Corinth Soft Soil Array (CORSSA), the location of which is shown in Figure 1 (Pitilakis et al., 2004). CORSSA is a vertical array consisting of five broadband 3D accelerometers, one at the surface and four at depths of 14 ,
31, 57, and $178 \mathrm{~m}$. All instruments are Kinemetrics Episensors (ES-T at the surface and ES-DH at all other depths) with a sampling rate of $200 \mathrm{~Hz}$ and a response that is practically flat up to a relatively high frequency (at least $60 \mathrm{~Hz}$ ). The soil profile at this location is known to consist of soft, loose materials down to $155-\mathrm{m}$ depth, which are underlain by a stiff conglomerate with $V_{S}>1000 \mathrm{~m} / \mathrm{s}$, and this in turn underlain by stiffer formations. The upper four instruments of the array are located within the soil formations, and the deepest (at $178 \mathrm{~m}$ ) is located in the conglomerate; in what follows we will refer to it as the "rock" station. The true seismic bedrock at this site, however, is a limestone lying at 700-m depth, as shown in the cross section of Figure 1 (Apostolidis et al., 2006).

The data set used here consists of 180 earthquakes recorded between 2002 and 2008. These were chosen from a larger data set compiled in Ktenidou (2010), which consisted of 520 events. The selection was based on the following criteria: depths small enough to ensure they took place within the crust (subduction events are used separately in a later section), good quality based on visual inspection, and the existence of an adequate pre-event noise record that allows the computation of the signal-to-noise ratio (SNR). All data used correspond to weak motion (PGA $<10$ gal for $95 \%$ of the events and $<90$ gal in all cases) and are thus unlikely to excite nonlinear responses in the soil column.

Figures 2 and 3 show the epicenter and magnitude distribution for the events. Moment magnitudes range from 1.9 to 6.5 , epicentral distances from 1 to $255 \mathrm{~km}$, and focal depths from 2 to $35 \mathrm{~km}$ (>90\% of the events have depths $<25 \mathrm{~km}$ ). The number of traces examined is 1800 : two components at five stations for 180 events.

The events used here were cross checked with the four major Greek earthquake catalogs to determine the earthquake parameters (see Data and Resources for details). The catalogs that were used are:

- Institute of Geodynamics, National Observatory of Athens (NOA): 2002-2008;

- Geophysical Laboratory Seismological Station, Aristotle University of Thessaloniki (AUTH): 2002-2007;

- Seismological Laboratory, National Kapodistrian University of Athens (NKUA): 2002-2008;

- Patras Seismological Laboratory (PSL, 2008): 2002-2004.

As there is no single official earthquake catalog for Greece, we compiled our catalog making use of all the existing ones. When one event was found in more than one catalog, the parameters were chosen from the catalog that was more complete (in the interest of relative homogeneity) and that offered the best network coverage. We noted some differences between catalogs not only in depth estimates but also in epicentral coordinates. In cross checking all catalogs, we detected all events for which large differences in location existed between catalogs; we compared them in terms of magnitude and origin time (allowing time differences up to 2-3 s) and eliminated any obvious bad correlations. For the remaining data, differences in event location between 
(a)

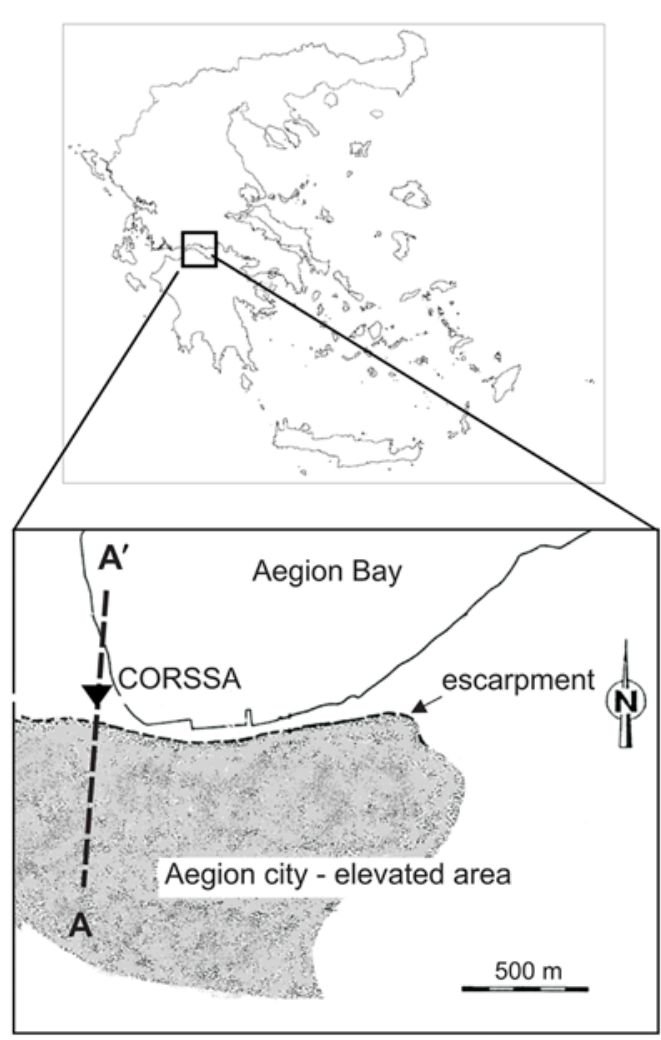

(b) $\mathrm{A}$

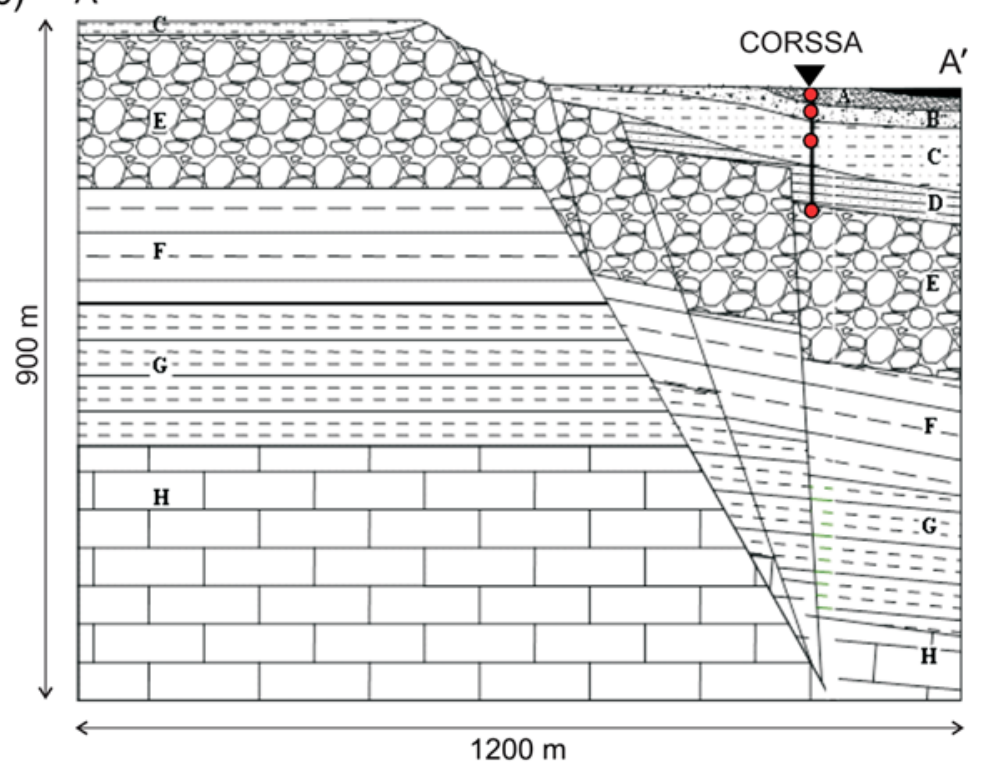

\begin{tabular}{c|cc|cc|c} 
Layer & Type & $\begin{array}{c}\text { Thickness } \\
(\mathrm{m})\end{array}$ & $\begin{array}{c}\text { Density } \\
\left(\mathrm{kN} / \mathrm{m}^{2}\right)\end{array}$ & Vs (m/s) & $\begin{array}{c}\text { Damping } \\
(\%)\end{array}$ \\
\hline A & Soft deposits & 20 & 18,5 & 180 & 1,8 \\
B & Soft deposits & 25 & 19,5 & 265 & 2,5 \\
C & Hard deposits & 55 & 19,5 & 440 & 2,7 \\
D & Very hard deposits & 55 & 20 & 540 & 2,0 \\
E & Conglomerate & & 21 & 1000 & 0,5
\end{tabular}

Figure 1. (a) Location of the city of Aegion, Gulf of Corinth. The locations of the Corinth Soft Soil Array, the fault escarpment, and cross-section A-A' are marked. (b) Cross-section perpendicular to the slope and table of soil properties at CORSSA, including layer thickness, soil density, $V_{S}$ velocity, and small-strain material damping (adapted with permission from Apostolidis et al., 2006). The color version of this figure is available only in the electronic edition.

catalogs may be due to various factors, including the assumptions made as to the velocity model, the software used for locating, or the layout of the network. The standard deviation for epicentral distances across catalogs is $\pm 5 \mathrm{~km}$, and for depths it is $\pm 7 \mathrm{~km}$.

\section{Preliminary Processing}

Acceleration time histories were baseline corrected and the horizontal components were rotated to the same system of axes for all five stations, namely perpendicular and parallel to the cross section of Figure 1 (this corresponds to an almost north-south [NS]least-west [EW] orientation). $P$ and $S$-wave arrival times were manually picked for each event on the rock records. $S$-wave windows were then extracted, the duration of which was first predefined based on magnitude and distance (ranging from $5 \mathrm{~s}$ for small, nearby events to $30 \mathrm{~s}$ for large, distant ones) and then visually adjusted, maintaining a minimum duration of $3 \mathrm{~s}$ in the interest of spectral resolution. Noise windows were also extracted, with a length that depended on the instrument pre-event memory. $S$-wave and noise windows were tapered at both edges with a Hanning taper (2.5\% of the window duration), then they were Fourier transformed and only the amplitudes of the spectra were kept.
The displacement spectra at depth were first plotted in $\log -\log$ space so as to pick the corner frequency for each event. Then the unsmoothed acceleration spectra were inspected in log-linear space and frequencies $f_{e}$ and $f_{x}$ were picked (following the nomenclature of Douglas et al., 2010), between which the decay is considered linear. Care was taken for this frequency range to be chosen to the right of the corner frequency so as to avoid any effects of the source. This is a precaution not always explicitly taken. Often $f_{e}$ may actually lie to the left of $f_{c}$. Tsai and Chen (2000) focus on the choice of $f_{e}$ and even criticize the seminal paper of Anderson and Hough (1984) for ignoring the importance of its choice. Another approach was proposed by Anderson and Humphrey (1991), where a joint computation of spectral level, $f_{c}$, and $\kappa$ is made and thus the picking of $f_{c}$ and check for $f_{e}$ is not necessary. In this study, however, we follow the classical method of Anderson and Hough (1984) where $f_{e}$ is handpicked and the spectra are only inverted for $\kappa$. Figure $4 \mathrm{a}$ shows the distribution of $f_{c}, f_{e}$, and $f_{x}$ with magnitude for all the events of our data set.

This manual picking was performed on each horizontal component for two stations of the array: the surface and the rock at $178 \mathrm{~m}$, as shown in Figure 4c, for example. To avoid further manual picking for the two components at the three 


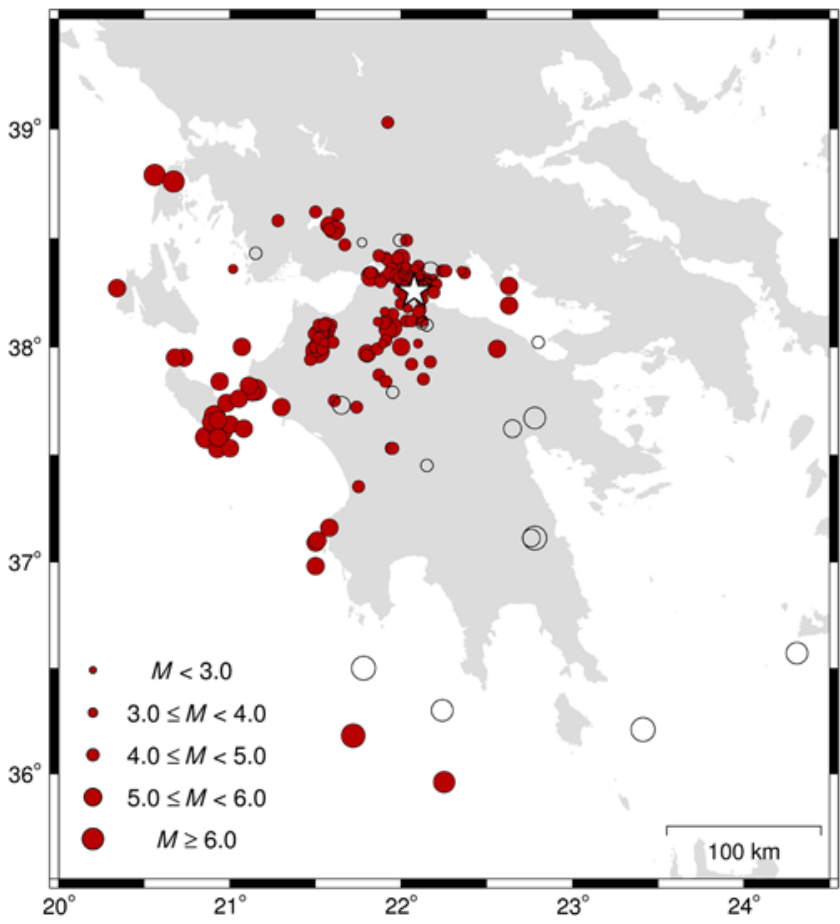

Figure 2. Epicenter distribution for the 180 crustal (solid circles) and 20 subduction (transparent circles) events of the data set. The size of the circle scales with moment magnitude. Location of CORSSA site is marked by a star. The color version of this figure is available only in the electronic edition.

intermediate stations (depths of 14,31 , and $57 \mathrm{~m}$ ), the manually picked frequency values at 0 and $178 \mathrm{~m}$ were used to fix a pair of $f_{e}-f_{x}$ values for each event separately. This pair of frequencies was then applied automatically to the remaining spectra. The resulting windows all passed through visual inspection to ensure that the spectral amplitude degradation in each of them was indeed linear.

Before proceeding with $\kappa$ computation using these extracted spectral windows, we check that only high-quality data are used. For this reason we set the strict requirement that the SNR must be above three for all spectra used. To

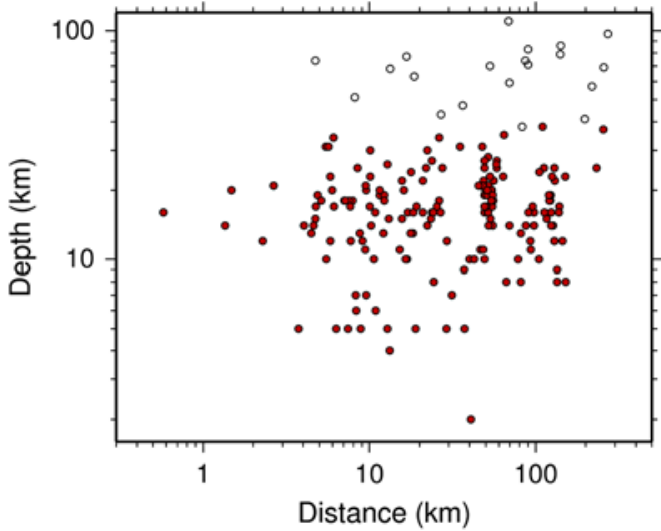

compute SNR we smooth the $S$ and noise window spectra with a mild Konno-Ohmachi filter for a bandwidth of $b=40$ (Konno and Ohmachi, 1998) and interpolate to a common frequency step and number of points to perform the spectral division. On the unsmoothed spectra, the frequency range $\Delta f=f_{e}-f_{x}$ was narrowed until the SNR inside it exceeded three. Spectra with ranges of $\Delta f<10 \mathrm{~Hz}$ were discarded in the interest of the robustness of the slope computation. Frequency $f_{e}$ ranges from 1 to $20 \mathrm{~Hz}$ and $f_{x}$ is between 11 and $70 \mathrm{~Hz}$, depending heavily on the magnitude and distance of the event (Fig. 4a). We may repeat here that the Nyquist frequency based on sampling rate is $100 \mathrm{~Hz}$ and that instrument response does not exhibit decay below $60 \mathrm{~Hz}$. This allows us to use a rather large range of frequencies for our $\kappa$ computations, an advantage which was not always a given in earlier studies.

\section{Computation of $\kappa$}

\section{Individual Event Values}

The aforementioned unsmoothed spectra were used to compute the linear trend of amplitude decay in the chosen frequency range. Initially, this was done for each event, depth, and component in two ways. The simplest way is to use standard linear regression, a standard procedure that seeks merely to minimize the sum of squared errors of all points from the line. The second is a more robust method of regression where the algorithm uses iteratively reweighted least squares with bisquare weighting. This method allows for results to be less influenced by outliers in the data, which in this case would mean any troughs and peaks that cause the spectral shape to deviate from its linear trend. It was found that outliers did not significantly affect the computation of the slope for the events studied, as the difference between the individual $\kappa$ values computed through robust and standard regression was $<8 \%$ for most events. We use the robust solution in what follows. The individual values of $\kappa$ were computed for each event, depth, and component based on the slopes of the spectra according to equation (4). The $\kappa$ values derived from the two

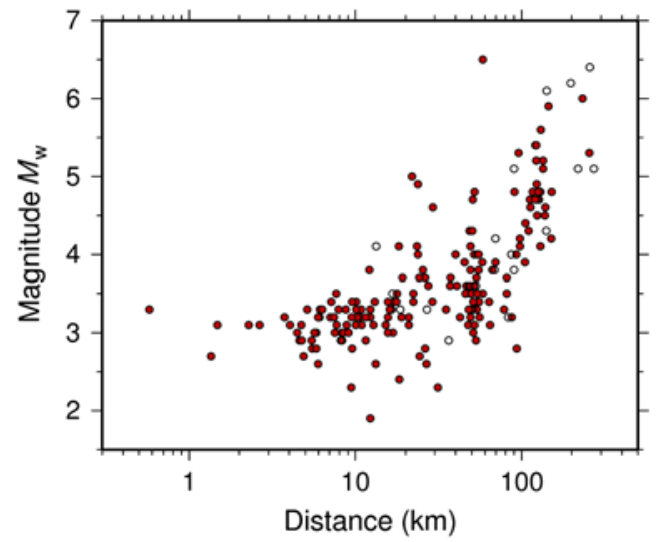

Figure 3. Magnitude and depth distribution with distance for the data set. The color version of this figure is available only in the electronic edition. 
(a)
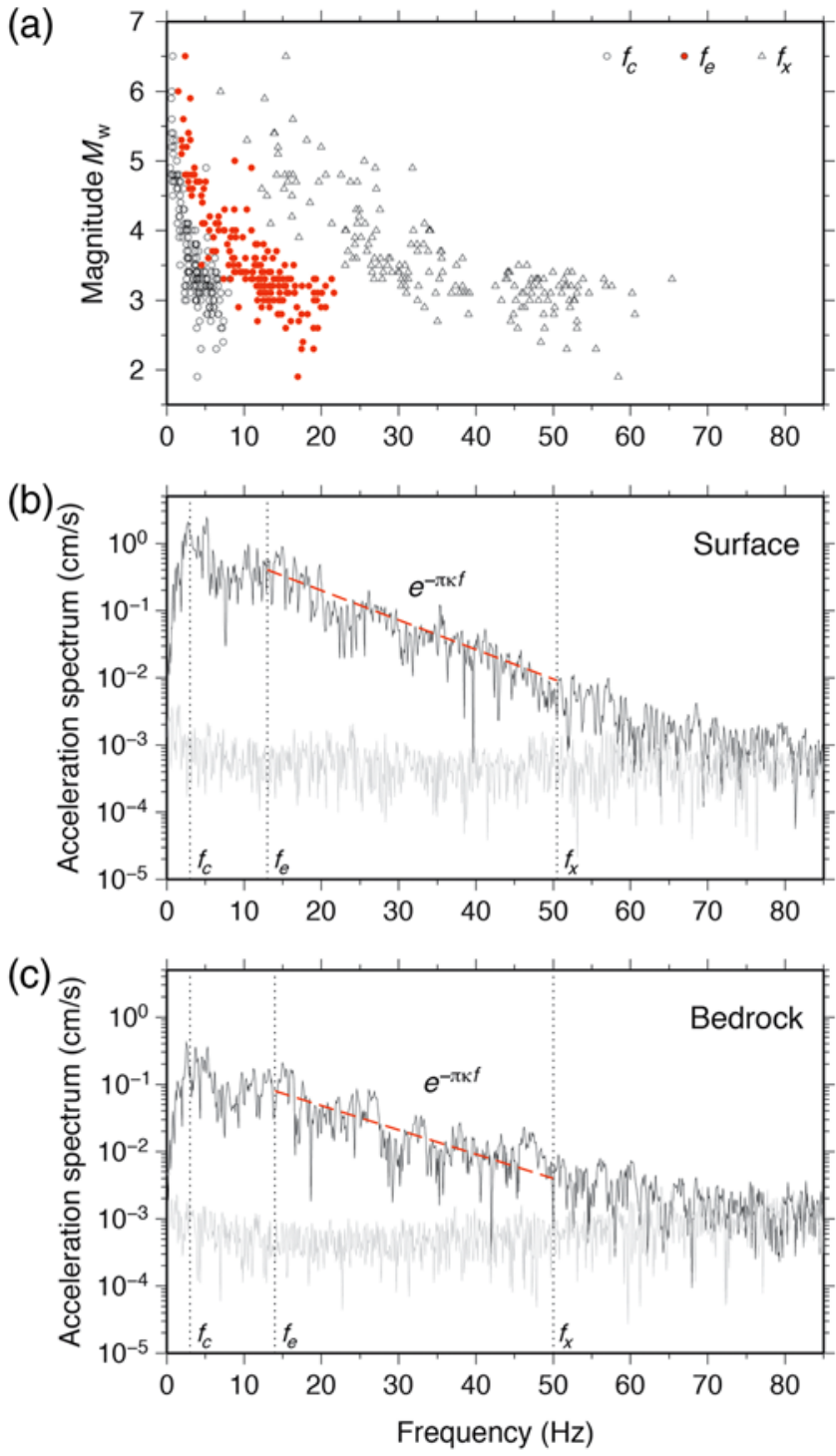

Figure 4. (a) The distribution of frequencies $f_{c}, f_{e}$, and $f_{x}$ for all events in the data set with respect to magnitude. (c) Example of the picking of $f_{e}$ and $f_{x}$ on the Fourier acceleration amplitude spectra for a record (b) at the surface and (c) at bedrock. Noise spectrum plotted in gray; $S$-window spectrum plotted in black. Corner frequency was read on the downhole rock displacement spectrum. The color version of this figure is available only in the electronic edition.

horizontal components were then combined into an average, yielding a single $\kappa$ value per event and station. The difference in $\kappa$ values between the two horizontal components was generally $<20 \%$, which is considered an acceptable limit by Van Houtte et al. (2011). We believe that average kappas computed from individual $\kappa$ values that are rather dissimilar (e.g., differences $>25 \%$ ) should not be used because large differences may be associated with numerical or waveform instability.

\section{Dependence on Distance}

Once the individual $\kappa$ values are computed, we can investigate their dependence on distance (equation 5). In the study that first introduced this concept, Anderson and Hough
(1984) used epicentral distance. In recent studies, however, there is no consensus as to which definition of distance to use. For instance, Douglas et al. (2010) use epicentral distances, whereas Castro et al. (2000) and Van Houtte et al. (2011) use hypocentral distances. The hypocentral distance may seem better associated to the path followed by the seismic waves from source to site, and thus more fit to describe the regional effect on $\kappa$. Its use, however, is accompanied by the added uncertainties of focal depth estimation. Furthermore, the main goal of $\kappa$ computation is to extrapolate the $\kappa(R)$ function to $R=0$ to estimate $\kappa$ at the site under study $\left(\kappa_{0}\right)$. We think this might be more convenient to do using epicentral distance, as hypocentral distance cannot equal zero unless event depth also does. Moreover, $\kappa$ was originally introduced with respect to epicentral distance by analogy with the problem of inverting travel times for the velocity in a layered Earth using ray tracing. If epicentral distance is replaced by hypocentral then the analogy is lost (J. Anderson, personal comm., 2012). On the basis of these arguments, we suggest the use of epicentral distance. Though physically $\kappa$ does not depend on the type of distance, in this study we present results for both types of distance and investigate the effects of this choice on the $\kappa$ estimate. We do not include here distance definitions related to the fault, though they are often used in applications such as GMPEs, because the necessary information is not always available and also because for large distances the difference would be small.

Figure 5 shows results for the surface station with respect to (a) epicentral and (b) hypocentral distances. It shows the regressed line plus/minus one standard deviation plotted onto the cloud of points. The regression parameters $\left(\kappa_{0}\right.$ and $\kappa_{R}$ of equation 5), the standard deviation, and the coefficient of correlation $\left(R^{2}\right)$ are also plotted. We see that the two types of distance yield similar slopes, $\kappa_{R}$, but different $\kappa_{0}$ values: $\kappa_{0}$ is lower if $R_{h}$ is used, because $R_{h}$ also includes the focal depth and thus shifts the computed best-fit line farther away from the $R=0$ axis on which $\kappa_{0}$ is measured. This difference is about $20 \%$ at the surface (and up to $40 \%$ at rock, not plotted here), which is significant and much larger than the standard deviation of the computed values. For this reason, we believe that the definition of distance is important and that when a certain estimate of $\kappa_{0}$ is used as input parameter (e.g., in GMPEs), it may be worth taking into account the type of distance used in its computation. This effect will be stronger the steeper the slope of the regressed line is, that is to say, the more attenuative the region (the lower the $Q$ ). Also, this effect could be stronger if subduction seismicity is studied, as the large depths would cause greater differences between epicentral and hypocentral distance values. Because of the inherent uncertainty in constraining the focal depth, we made an additional set of regressions for hypocentral distance, assuming all events to have a common depth of $15 \mathrm{~km}$. The difference between $\kappa_{0}$ from this approach and from case (b) is no more than $2 \%-5 \%$ for robust and standard regression, respectively. This shows that the choice between epicentral and hypocentral distance is important for this data 

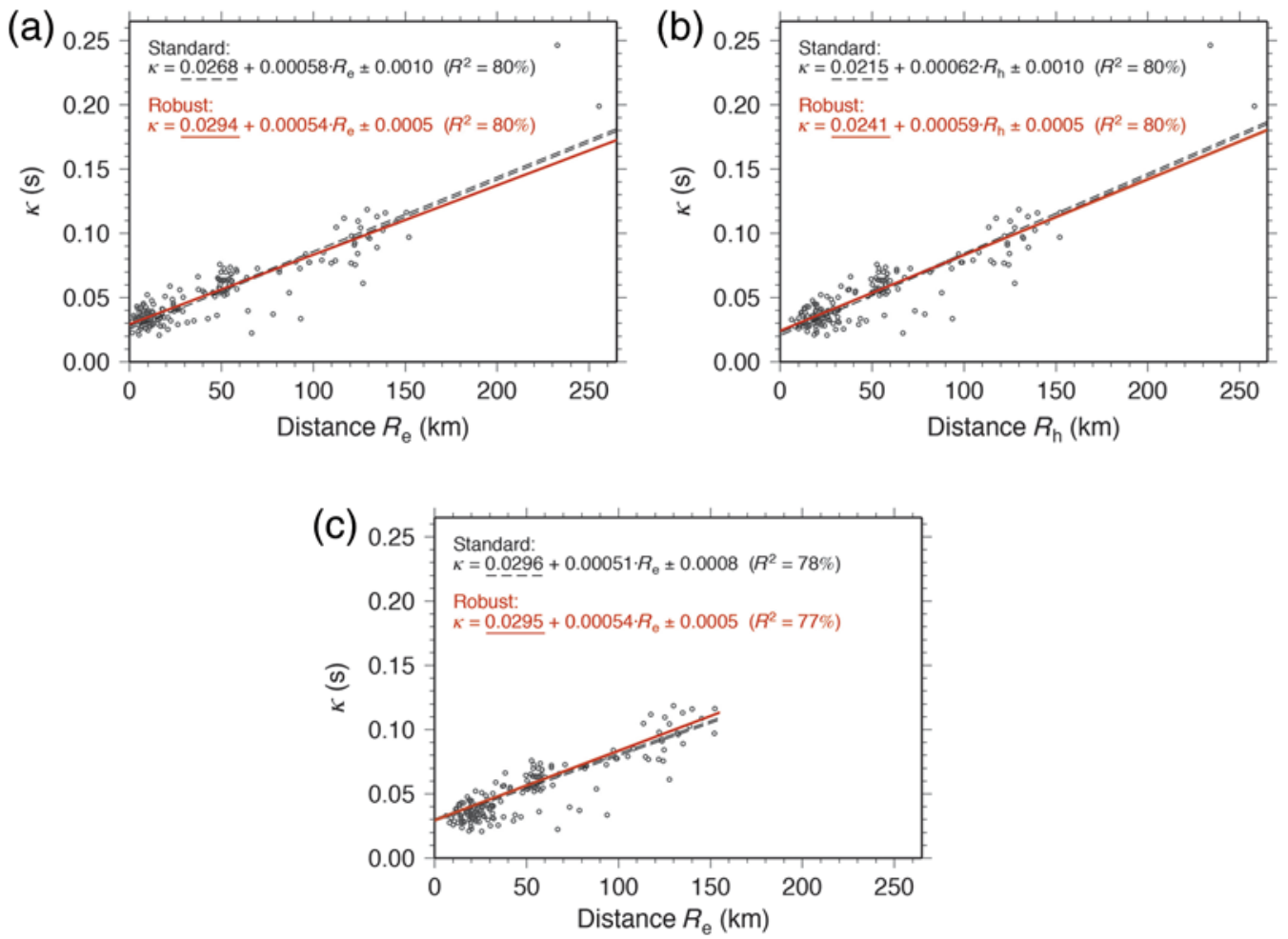

Figure 5. Distribution of individual $\kappa$ values (180 events, average values for the two horizontal components) computed at the surface station of CORSSA. Regressed lines (average \pm 1 standard deviation) resulting from standard linear (dashed) and weighted robust (solid) individual regressions are plotted onto the data points. Formulas show average line equations, standard deviation, and coefficient of correlation. Computations made with (a) epicentral and (b) hypocentral distance yield systematically larger $\kappa_{0}$ values for $R_{e}$. (c) Individual $\kappa$ values at the surface station of CORSSA with epicentral distance, only for events up to $150 \mathrm{~km}$. The color version of this figure is available only in the electronic edition.

set irrespective of the quality of depth estimation and even for relatively shallow seismicity.

A second issue to consider is the type of dependence on distance. Most studies to date assume a linear dependence of $\kappa$ for simplicity. In our case we adopted the model of equation (5) first based on visual inspection. After regressing our data we find a coefficient of correlation of $80 \%$ (Fig. 5a), which indicates that a linear model is adequate for this data set.

Note that we do not focus on the dependence of $\kappa$ with source (as did, e.g., Purvance and Anderson, 2003), but only with site and distance. We had no information as to the focal mechanisms (other than that in this region faults are normal) or fault-plane solutions. As to magnitude, a posteriori comparisons showed no correlation between it and our computed $\kappa$ values. Recent work (Kilb et al., 2012) shows that $\kappa$ estimates based on magnitudes even smaller than 1.0 can match values for $M>3.5$.

\section{Regression with Distance}

Another issue we raise concerns the type of regression performed. As we did for individual event values, here again we perform two types of regression: the standard linear regression and the robust linear regression that helps minimize bias from outliers. Outliers here are mostly data at large dis- tances where the scatter is larger and the absolute values themselves are also larger. In the case at hand, the data set is complete in terms of distances up to $150 \mathrm{~km}$; after that, the data is very sparse (only two points at distances more than $200 \mathrm{~km}$ ). In Figure 5c we show the importance of the type of regression in combination with the completeness of the data set: when using the entire data set (Fig. 5a), the standard regression tends to bring the line nearer to these two points, whereas the robust regression maintains it nearer to the overall average. When excluding the two outliers (Fig. 5c), the two approaches give more similar results, as seen by the equations. Moreover, the results of the robust approach with or without the outliers are very similar. Thus, the robust approach permits us to use the few records we have at large distances without allowing them to bias the overall results. As seen from the figure, the use of standard regression affects the slope of the line, drawing it upwards at large distances. The result is that the intercept with $R=0$ is forced downwards. This is reflected in the equations shown, where $\kappa_{0}$ is underestimated by $10 \%$ due to the outliers. Finally, as seen from the equations, when using the robust approach the standard deviation is reduced by $50 \%$. Based on the better stability, we suggest that if the data set used is not complete in terms of distance (which is usually the case) or if there are important outliers (owing perhaps to the spatial variation of 
$Q)$, the use of the simple regression may incur bias. We note here that the robust regression may not be as necessary if the individual points could be regressed taking into account their error bars. This is done in Douglas et al. (2010), where the error bars derive from the fact that three different analysts made the picking of the $f_{e}-f_{x}$ frequency range so as to resolve special cases where $\kappa$ values are very sensitive to the picking. In our case, as the picking was made by a single person, we have no way of estimating this uncertainty. Thus, the robust approach may alleviate any such bias in the process.

\section{Choice of Data Set}

\section{Data Set Orientation}

A logical question that arises is whether the orientation of the data set, that is to say, the azimuthal distribution of the epicenters, may have an effect on the results. This question might stem both from the sharp lateral variation in surface geology that is observed locally at Aegion (Fig. 1), as well as from possible lateral variation of $Q$ in the wider region covered by the ray paths studied. To investigate this we divide the data set into groups according to azimuth. Figure $6 \mathrm{a}$ shows the epicenters of these groups along with their computed values of $\kappa$ (Fig. 6b). We note there is no systematic behavior with respect to where the events come from. We use a solid ellipse to highlight the behavior of a particular cluster of events occurring near the island of Zakynthos. Despite their close epicenters, the corresponding $\kappa$ values show a significant scatter. Castro et al. (2000) attributed such effects to uneven attenuation near the source. We also use a dashed circle in the figure to highlight the behavior of a group of events that occur at the same distance from the site $(\sim 50 \mathrm{~km})$ but the epicenters of which are distributed in different directions around it. The $\kappa$ values of these events are very similar despite the geographic distribution and the variety of source-site geometries, showing no clear azimuthal tendencies.

\section{Type of Seismicity}

We compare the results derived so far from our data set of 180 crustal earthquakes with those of 20 deep events that originated in the subduction zone and whose paths also sample the mantle. In separating the two groups, we consider as a limit value the depth of $35 \mathrm{~km}$. Papazachos and Nolet (1997) found crust thickness in Greece to range mostly between 30 and $40 \mathrm{~km}$, whereas the New Global Crustal Model CRUST2.0 (Bassin et al., 2000) indicates crustal depths up to $35 \mathrm{~km}$. Given the uncertainty in depth estimation, to decide the type of seismicity, we used depth estimates from all catalogs where available; we considered as subduction events those with depths $>35 \mathrm{~km}$ in most catalogs. These events have moment magnitudes between 2.9 and 6.4, depths from 40 to $110 \mathrm{~km}$, and epicentral distances from 5 to $272 \mathrm{~km}$ (shown as transparent circles in Fig. 2). In Figure 7 we compare $\kappa$ values for subduction events (large circles) with the results derived for the crustal data set (small circles). We do not have enough subduction data to propose a $\kappa$ model for this type of event, nor is it certain that such a model should follow equation (5). We make a tentative regression for these points, however, and find that the slope is milder than for crustal events and the intercept is smaller. This was also found by Van Houtte et al. (2011) for Japan and may be due to higher $Q$ values in mantle and deep crustal paths. Subduction data also present considerable scatter, possibly due to large spatial variability in deeper $Q$.

\section{Site Resonance Correction}

Up to now we have not corrected the records for resonance effects. At CORSSA, the fundamental frequency corresponding to the sediment-conglomerate interface at $155-\mathrm{m}$ depth is $0.9 \mathrm{~Hz}$, and the first few harmonics are observed beneath $10 \mathrm{~Hz}$. The frequency range we used to derive $\kappa$ is for most events between 10 and $60 \mathrm{~Hz}$, that is, it is rather wide and above the first resonant peaks. Thus, according to Parolai and Bindi (2004), resonance effects should not inhibit the estimation of $\kappa$ at the surface.

We attempt the site effect correction driven by a different motivation, however. At this site we have both surface and downhole records, which allows us to directly compute $\kappa$ at both levels. We also have a good knowledge of the soil profile at the site thanks to the model of Apostolidis et al. (2006). This allows us to investigate whether it may be possible to deconvolve the theoretical transfer function computed on the 1D profile (see model properties in Fig. 1) from surface motion to compute $\kappa$ at rock level. This may be of interest in cases where only surface stations are available (i.e., most cases) and $\kappa$ needs to be defined at rock level.

Using Kennet's (1983) reflectivity method we compute the theoretical 1D transfer function between the surface and the conglomerate rock in which the deepest station lies, based on a local 1D model extracted from the 2D model of Figure 1. With this transfer function we deconvolve the surface accelerograms to rock level. Using these corrected accelerograms we repick frequencies $f_{e}$ and $f_{x}$, recompute individual $\kappa$ values, and regress to get a $\kappa$ model as a function of distance. The $\kappa$ models for the deconvolved motion are compared to those for the (uncorrected) surface and downhole records in Figure 8a. We find that after the correction the values of $\kappa_{0}$ decrease by $20 \%-50 \%$ (depending on the type of distance and regression) below the $\kappa_{0}$ values of the rock records. The model derived from the deconvolution seriously underestimates $\kappa$ at all distances and provides, at best, a lower bound for the values observed at downhole rock.

One possible interpretation for the discrepancy between downhole and deconvolved surface $\kappa$ estimates would be to assume that the station at $178 \mathrm{~m}$ is not an adequate reference station and is affected by downgoing waves; thus, its response still includes site effects and reverberations that increase $\kappa$. In previous instrumental and numerical studies of site amplification at CORSSA (Ktenidou, 2010; Ktenidou et al., 2011), 
(a)

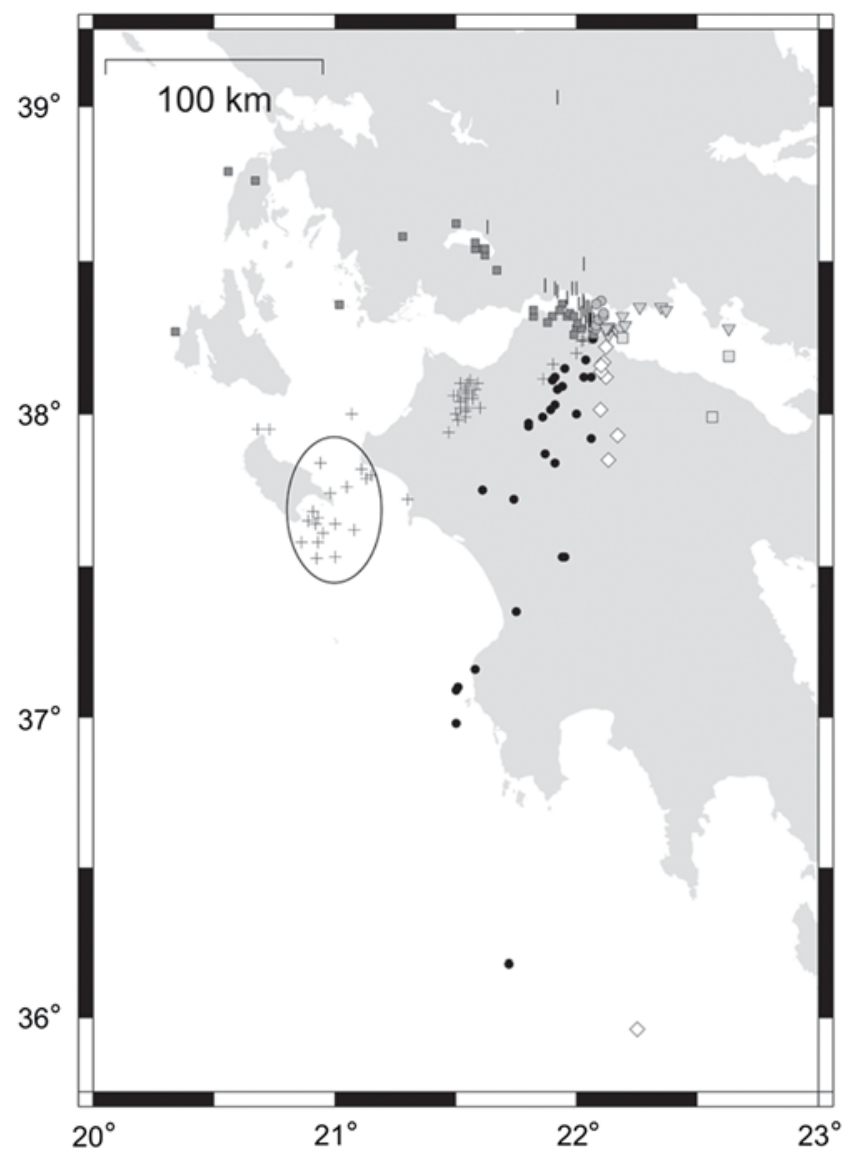

(b)

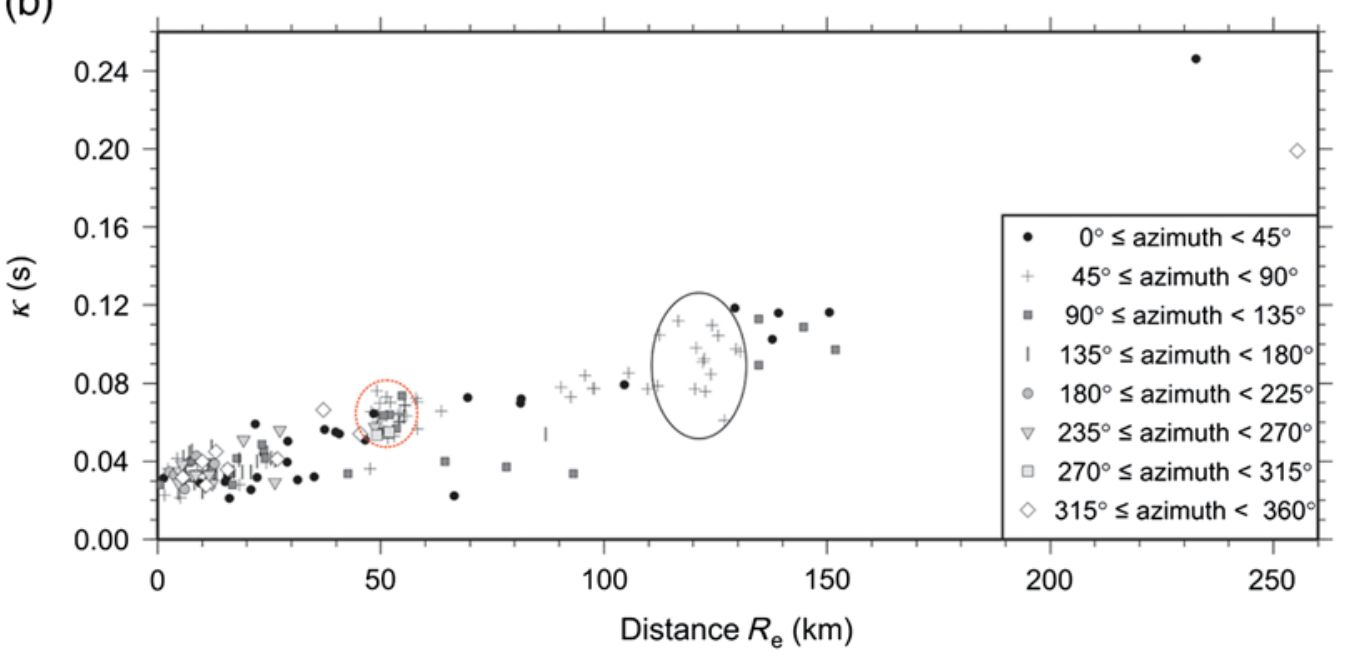

Figure 6. Grouping of events according to azimuth: (a) epicenter distribution and (b) $\kappa$ value distribution. Ellipses indicate the scatter in the results for a particular cluster of events near Zakynthos Island. The circle indicates that results for events occurring at a distance of $50 \mathrm{~km}$ around the site from various different directions cannot be distinguished according to azimuth. The color version of this figure is available only in the electronic edition.

however, we found that though there is a reflected wave field at the downhole station, it is relatively weak. It causes a slight trough in the transfer function at the fundamental frequency and some fluctuations due to destructive interference, but the horizontal-to-vertical empirical spectral ratios (HVSR) remain relatively flat and very near unity, with values ranging from
0.7 to 1.5 , up to $100 \mathrm{~Hz}$ (a relatively flat HVSR near unity has been considered to indicate the lack of site effects at a reference station, e.g., Raptakis et al., 2005). Furthermore, as in the case with reverberations near the surface, the Parolai and Bindi (2004) rule of thumb may apply also to downgoing wave contamination: namely, that if a wide enough frequency 


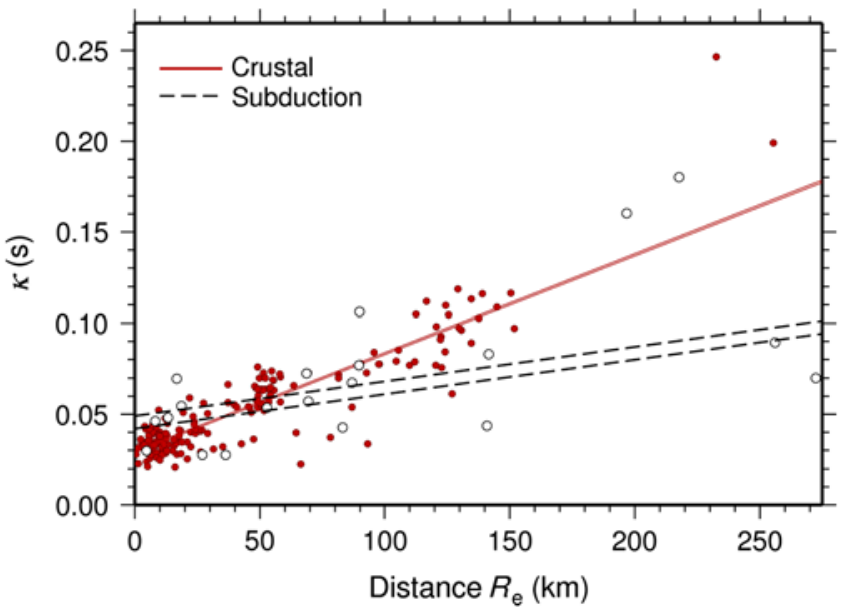

Figure 7. Individual $\kappa$ values at the surface and regressed lines ( \pm 1 standard deviation) from robust regression for crustal (solid lines and dark circles) and subduction (dashed lines and open circles) seismicity. The color version of this figure is available only in the electronic edition.

range is chosen far enough from the frequencies amplifiedor, here, deamplified - the reverberations should not affect $\kappa$ seriously. As an indication of amplification, in Figure 8c and $8 \mathrm{~d}$ we plot acceleration time histories and Fourier spectra at all stations for a single event (the one shown in Fig. 4).

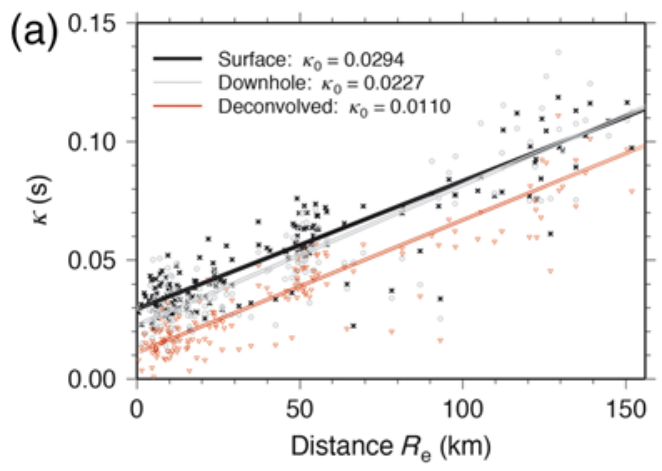

(c)

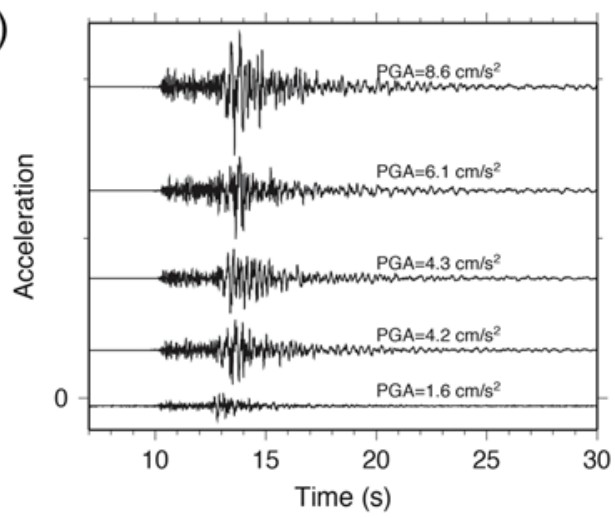

Another more probable explanation for the observed discrepancy is that the theoretical transfer function may not be dependable at very high frequencies. Though it is comparable to empirical transfer functions previously computed at CORSSA using standard spectral ratios (SSRs) and HVSRs, this similarity can only be verified at relatively low frequencies (Fig. 8b), especially as the site is soft and our 1D model does not account for very fine surface structure (its surface layer has a thickness on the order of $20 \mathrm{~m}$ ). Thus, we view theoretical results at very high frequencies (e.g., between 20 and $50 \mathrm{~Hz}$, which is the range for which $\kappa$ is picked for most records) with caution, as we cannot be sure they are free of artifacts.

Few studies correct surface records for site amplification (e.g., Margaris and Boore, 1998) before computing $\kappa$. Douglas et al. (2010) did not correct accelerograms for site response in the interest of simplicity. Van Houtte et al. (2011) attempted correction of surface records but did not implement it in their final results because the transfer function introduced problems at high frequencies. According to them, site effects were visible in the results through the greater variability in $\kappa$ values at the surface compared to the downhole values. Overall, it is not often that $\kappa$ is computed from any records other than surface records, and those will inevitably contain some resonance effects. We see here that, even in
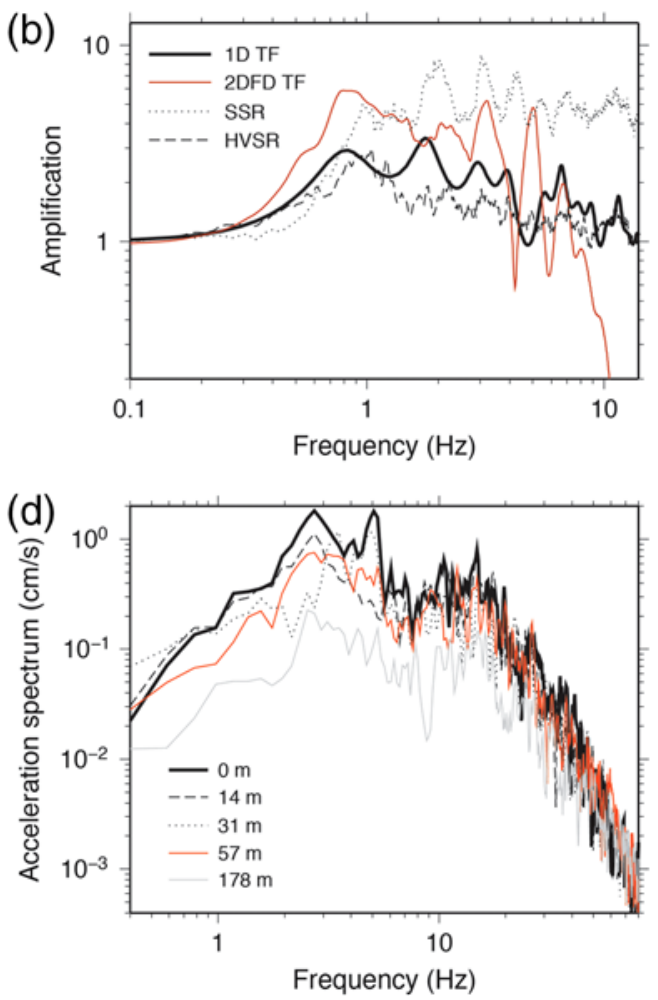

Figure 8. (a) Data points and regressed lines ( \pm 1 standard deviation) resulting from weighted robust individual regressions with epicentral distance, computed for the uncorrected surface records, the downhole records, and the surface records after deconvolution to rock with the 1D theoretical transfer function. (b) 1D theoretical transfer function (thick solid line), 2D numerical transfer function derived from finite different analysis (thin solid line), and empirical spectral ratios SSR (dotted line) and HVSR (dashed line), computed by Ktenidou (2010). (c) An example of acceleration histories at all stations of the array, showing the effect of amplification for the same event depicted in Figure 4. (d) An example of $S$-wave spectra at all stations of the array. The color version of this figure is available only in the electronic edition. 
such a case as ours where the profile and soil properties are well known and the theoretical transfer function can also be verified through empirical data, using the transfer function for deconvolution (e.g., in order to arrive at rock level motion) is not necessarily a straightforward task. It may add to the uncertainty by introducing more errors than it takes away, or rather, introducing them in the range of frequencies that matters most.

\section{Effect of $V_{S}$ and Data Redundancy}

Above, we observed and compared results for the topmost and deepest stations. Table 1 shows results (regression parameters and standard deviation) for all five stations, using robust regressions and epicentral distance. Though the values of $\kappa_{0}$ at each station correlate as expected with the $V_{S}$ of each layer (the former decrease as the latter increase), we do not obtain the same slope value for all the stations. The slope is similar for the four stations that lie in soil, but it is steeper for the rock (i.e., 178-m depth). Based on the hypothesis that the slope reflects regional effects, it should be common for all five stations. It is not the first incidence of such a result. Hough et al. (1988) compared the variation of $\kappa$ between a deep sediment site and a granite site (fig. 5 of that article). They concluded that although $\kappa_{0}$ values differed greatly between the two sites, the slopes seemed by inspection similar. Based on this observation, they then considered $\kappa_{R}$ a regional characteristic and $\kappa_{0}$ a reflection of the local structure a few kilometers below the station. Looking more closely at that figure, however, a linear regression would allow different $\kappa_{R}$ values. In another study, Anderson and Hough (1984) computed $\kappa$ models for three types of sites: alluvium, consolidated sediments, and hard rock. $\kappa_{0}$ were similar for the first two types and decreased for the third one (similar to our observations). They also found different $\kappa_{R}$ values among the site types. These authors, however, considered the difference in slope negligible with regard to the scatter in their data and thus concluded that there is no dependence of the slope on site type. This gives rise to the question of how data from different stations should be treated, and in particular, such differences in slope. Although in earlier times the limited amount of data may have yielded a scatter large enough to justify ignoring such differences, in future studies the volume of data may decrease the scatter and the question may arise of whether such differences are statistically significant. In our case, given the computed confidence intervals, we consider the difference significant. Thus, to be consistent with the physics behind the model we are using, we will investigate ways to achieve a common slope for all data to represent the regional effect.

We are not aware of many studies that look into $\kappa$ beneath the surface. Thus, this kind of problem has not often been faced before. Douglas et al. (2010) made a multiple regression using data recorded on soil and rock outcrop, constraining the slope to remain constant for both site types, based on the idea of Anderson (1991) that the slope is common for all stations in the region. Van Houtte et al. (2011), on the other hand, who used data from the Japanese KiK-net network, considered that the slope is best computed based only on downhole rock data, so they computed it at depth and then fixed it for the regression at the surface. As alternative solutions to the independent regressions, we recompute $\kappa$ according to these two strategies of constraining the slope. First, we make a multiple regression using data from all stations at the same time and constraining the slope to remain constant at all depths. Then we make individual regressions at each station in soil after fixing $\kappa_{R}$ to its value at depth. In Tables 2 and 3 we show the results according to these two assumptions, respectively. We find that $\kappa$ estimates depend not only on such factors as the definition of distance or the type of regression but also on the data available and the way they are combined, for example, which stations are used in the regressions and how. If we only had surface data available, the computations would be more straightforward and we would not have seen this variability.

To understand the reason for this change in slope between soil and rock stations we return to the data and inspect

Table 1

Results of Robust Regressions with $R_{e}$ Using Individual Independent Regressions at All Stations

\begin{tabular}{rrcccc}
\hline Station & $V_{S}(\mathrm{~m} / \mathrm{s})$ & $\kappa_{0}(\mathrm{~s})$ & $\kappa_{R}(\mathrm{~s} / \mathrm{km})$ & $\mathrm{SD}(\mathrm{s})$ & $R^{2}$ \\
\hline $0 \mathrm{~m}$ & 180 & 0.0294 & 0.00054 & 0.0005 & $80 \%$ \\
$14 \mathrm{~m}$ & 265 & 0.0296 & 0.00054 & 0.0005 & $77 \%$ \\
$31 \mathrm{~m}$ & 440 & 0.0268 & 0.00055 & 0.0005 & $81 \%$ \\
$57 \mathrm{~m}$ & 540 & 0.0226 & 0.00056 & 0.0004 & $83 \%$ \\
$178 \mathrm{~m}$ & 1000 & 0.0226 & 0.00059 & 0.0006 & $80 \%$ \\
\hline
\end{tabular}

Table 2

Results of Robust Regressions with $R_{e}$ to Achieve a Common Slope, Using Simultaneous Multiple Regression with Contributions from All Stations

\begin{tabular}{rccccc}
\hline Station & $V_{S}(\mathrm{~m} / \mathrm{s})$ & $\kappa_{0}(\mathrm{~s})$ & $\kappa_{R}(\mathrm{~s} / \mathrm{km})$ & $\mathrm{SD}(\mathrm{s})$ & $R^{2}$ \\
\hline $0 \mathrm{~m}$ & 180 & 0.0293 & 0.00055 & 0.0005 & $95 \%$ \\
$14 \mathrm{~m}$ & 265 & 0.0275 & 0.00055 & 0.0005 & $95 \%$ \\
$31 \mathrm{~m}$ & 440 & 0.0265 & 0.00055 & 0.0005 & $95 \%$ \\
$57 \mathrm{~m}$ & 540 & 0.0230 & 0.00055 & 0.0005 & $96 \%$ \\
$178 \mathrm{~m}$ & 1000 & 0.0239 & 0.00055 & 0.0005 & $96 \%$ \\
\hline
\end{tabular}

Table 3

Results of Robust Regressions with $R_{e}$ Using Individual Regressions with Slope Fixed at Rock Value

\begin{tabular}{rccccc}
\hline Station & $V_{S}(\mathrm{~m} / \mathrm{s})$ & $\kappa_{0}(\mathrm{~s})$ & $\kappa_{R}(\mathrm{~s} / \mathrm{km})$ & $\mathrm{SD}(\mathrm{s})$ & $R^{2}$ \\
\hline $0 \mathrm{~m}$ & 180 & 0.0281 & 0.00059 & 0.0001 & $96 \%$ \\
$14 \mathrm{~m}$ & 265 & 0.0262 & 0.00059 & 0.0001 & $96 \%$ \\
$31 \mathrm{~m}$ & 440 & 0.0253 & 0.00059 & 0.0001 & $94 \%$ \\
$57 \mathrm{~m}$ & 540 & 0.0218 & 0.00059 & 0.0001 & $96 \%$ \\
$178 \mathrm{~m}$ & 1000 & 0.0226 & 0.00059 & 0.0001 & $95 \%$ \\
& & & & & \\
\hline
\end{tabular}


(a)

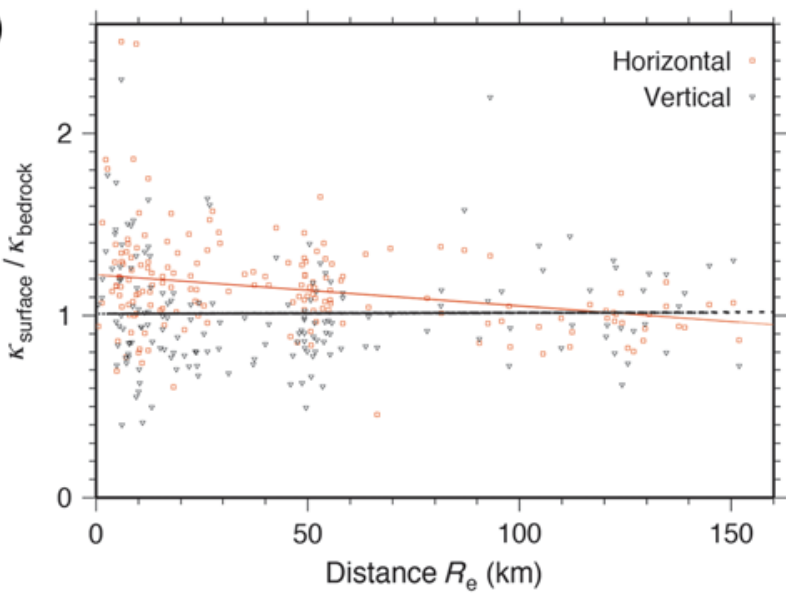

(b)

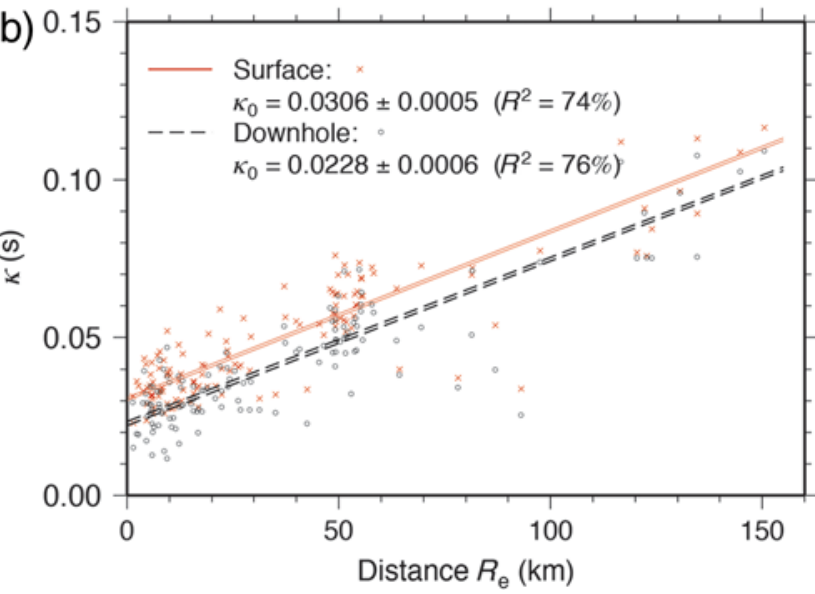

Figure 9. (a) The dependence on distance of individual $\kappa$ values and the quantity $\kappa_{0 \mathrm{~m}} / \kappa_{178 \mathrm{~m}}$ for the horizontal component (circles) and the vertical (triangles). (b) Individual $\kappa$ values for the subset of 128 events for which $\kappa_{0 \mathrm{~m}}>\kappa_{178 \mathrm{~m}}$ at the surface and deepest station of CORSSA with epicentral distance. Regressed lines (average \pm 1 standard deviation) resulting from robust individual regressions are plotted. Formulas show average line equations, standard deviation, and coefficient of correlation. The color version of this figure is available only in the electronic edition.

the individual values of $\kappa$ at various distances. The intersection at $R_{e}=100 \mathrm{~km}$ of the two $\kappa$ models based on the surface and the downhole data seen in Figure 8a (rather than the lines being parallel) means a different increase of the individual $\kappa$ values with distance for the two stations. Figure 9a shows the dependence with distance of the ratio of the individual values, that is, the quantity: $\kappa_{0 \mathrm{~m}} / \kappa_{178 \mathrm{~m}}$. We find that for the horizontal components (open circles) this ratio is indeed a function of distance. At relatively short distances (up to $100 \mathrm{~km}$ ), $\kappa$ is generally larger at the surface than at depth, whereas for larger distances the situation is almost reversed. This effect has not been reported before. Both by intuition and based on the literature, it would be expected that $\kappa$ values be systematically higher at the surface than at depth, thus correlating with $V_{S}$. Not many studies compare surface and downhole results. Oth et al. (2011) used data from the Japanese K-NET and KiK-net networks and computed $\kappa$ values at the surface and at depth to account for attenuation differences in source spectra at different depths due to site effects, after having corrected for path attenuation. They found that $\kappa$ values at the surface were on average larger than those at depth. To our knowledge, only Van Houtte et al. (2011) have proposed complete $\kappa$ models (i.e., as functions of distance, following the classical AndersonHough method) for both surface and downhole stations.

At this point, note that Van Houtte et al. (2011) used what they called a "performance criterion" in choosing their data set: they only accepted events that produced larger $\kappa$ values at the surface with respect to the borehole. This means that they did not use any points that in Figure 9a would plot beneath 1 . This gives a third possible alternative solution to the problem we face regarding the slope. We apply this criterion to our data set and choose a subset of 128 events for which $\kappa_{0 \mathrm{~m}} / \kappa_{178 \mathrm{~m}}>1$. We recompute $\kappa$ as a function of distance and plot the results in Figure 9b. This has an effect on the data set completeness with distance, as relatively few data points are left at longer distances to constrain the slope (as seen comparing with Fig. 8a). This causes the decrease in the coefficient of correlation in the new regression results. The slope becomes less steep for the downhole results, however, and thus the counterintuitive observations are now corrected, with the slope being the same at both stations and all $\kappa$ values correlating with $V_{S} . \kappa_{0}$ values for this approach are similar to those for the multiple regression. The excluded events do not share any common characteristics, such as magnitude, depth, or distance, so it would not have been possible to exclude them a priori. We do not know if the agreement between the two alternative methods- the multiple regression that constrains the slope and the $\kappa_{0 \mathrm{~m}} / \kappa_{178 \mathrm{~m}}>1$ constraint-is specific to this data set, but we prefer the former solution. It allows us to interfere less with the data set and is more practical in view of the scatter of each individual $\kappa$ value; even more so if we have numerous stations, as is the case here: if we had wanted to do this for all five stations rather than for just two (surface and rock), it would be almost impossible to compare values among the five stations and keep only those for which kappas is strictly ordered as expected. Moreover, the number of events and the distance coverage are not always rich enough to afford eliminating nonconforming data points.

\section{Effects of Other Parameters}

In this section, we briefly mention some further parameters that affect the computation of $\kappa$.

First we examine the SNR. In Preliminary Processing, we mention that after picking frequencies $f_{e}$ and $f_{x}$ on the unsmoothed spectra, those are then smoothed, the SNR is computed, and only the part for which it is higher than 3 is used to make the regressions for the individual $\kappa$ values. To see if the choice of SNR can affect our results, we experiment with values from 1 to 3 , recomputing individual kappas and $\kappa$ models with distance. We find a slight decrease of $\kappa_{0}$ and a 
slight increase of $\kappa_{R}$ as the SNR criterion becomes stricter. This can be explained as follows. When the $f_{e}-f_{x}$ frequency range is manually picked, it may extend to high frequencies where the noise level is high. If we consider a low SNR value (e.g., 1), then we will allow $f_{x}$ to lie within the noise, where the slope is actually near zero, whereas it should really have been picked farther to the left; such an error will cause the regression in the $f_{e}-f_{x}$ range to yield a lower value for the slope (and thus the individual $\kappa$ value) than the actual one. Such errors are easier to make for larger and more distant events where noise starts to contaminate signals at lower frequencies. Thus, individual $\kappa$ values will be underestimated mainly at larger distances, which in turn control the slope in the final regression with distance. Thus, the regression will yield a higher intercept $\left(\kappa_{0}\right)$ and lower slope $\left(\kappa_{R}\right)$. A strict SNR criterion, on the other hand, will force $f_{x}$ to the left, thus making sure that the regression is made in a range where the amplitude decay is indeed linear. A higher SNR value, then, may help remove bias due to user subjectivity. SNR as a quality criterion is not used by all authors computing $\kappa$, and when it is, it is not always used in the same way. In the few studies that mention SNR explicitly, values range from 1 to 5 . We believe it is important to make an SNR check. Furthermore, a value below 2 may be too low to study $S$ waves, given that values of 1 to 2 are often used in certain applications to define coda windows. In addition, the signal window is in reality a signal-plus-noise window, so SNR $>1$ does not even guarantee that the pure signal is higher than the noise. Thus, we believe that SNR is another of the factors that may add to the variability of $\kappa$ across literature and suggest that higher SNR values may lead to safer estimates.

Another parameter we examine is the choice of the system of horizontal axes. The final regression with distance is performed on the individual $\kappa$ values after they have been averaged for the two horizontal components. We investigated whether the orientation of these components affects $\kappa_{0}$. The reference system of axes here is defined with respect to the site's geomorphological features. We recomputed $\kappa$ using a different orientation of horizontal components and found that $\kappa_{0}$ values may vary up to $5 \%-10 \%$. There is no way to control this variability as the orientation does not affect the regression process in a systematic way. It may be that most of $\kappa$ is from local scattering that homogenizes the wave field with respect to back azimuth.

We also mention here a point already made by other researchers (e.g., Castro et al., 2000; Douglas et al., 2010) that is not always rigorously adhered to: the importance of keeping to the right of the corner frequency when picking $f_{e}$. Even with a careful visual inspection of the acceleration spectra, it is possible to introduce bias by picking $f_{e}$ for frequencies that are too low, for example, well within the plateau of the displacement spectrum. This adds source effects to the computation of a parameter that we accept here as related to site effects. For our data set, a preliminary picking of $f_{e}$ made by careful visual inspection but without regard to

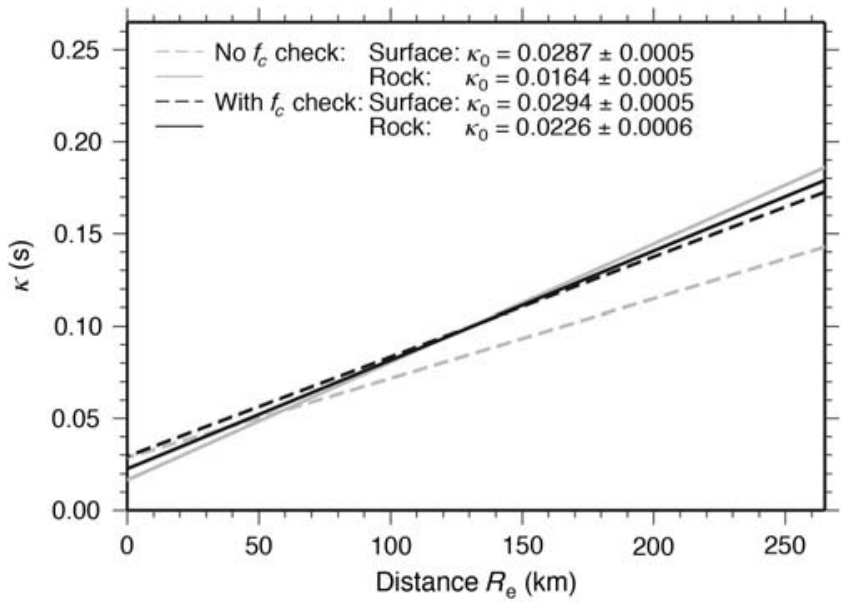

Figure 10. Data points and regressed lines $( \pm 1$ standard deviation) resulting from weighted robust individual regressions with epicentral distance, computed at the surface and at depth with and without explicitly checking to avoid the corner frequency.

$f_{c}$ affected $\kappa_{0}$ values up to $30 \%$. The results are shown in Figure 10.

Finally, given that we had to compile a "best catalog" out of the four existing earthquake catalogs, we also compute $\kappa_{0}$ treating these separately so as to see the sensitivity of the regression results to the location given by each one (for the computed regressions (E) see Fig. S1 of the electronic supplement to this article). The results are significantly different only if the least complete catalog is used on its own, which means that our choice of catalog in this case is important not so much due to differences in event location but rather due to completeness.

\section{The Vertical Component}

Few studies compute $\kappa$ on the vertical component of motion, though it may be interesting, for example, in the case of 1D instruments. Douglas et al. (2010) compute individual $\kappa$ values for the vertical component as well as for the horizontals (see fig. 4 of that article) but do not use them to create $\kappa$ models with distance. They conclude that individual $\kappa$ values are slightly lower for the vertical but generally comparable, so that in the absence of the horizontals they might be used to provide an initial estimate, possibly after a small adjustment. In Figure 11, we compare the individual $\kappa$ values at the surface and at the deepest station of CORSSA. Based on their similar scatter around the diagonal, results at depth (circles) are similar in the two directions. At the surface, however (dashes), the correlation depends on the absolute value of $\kappa$, which in turn depends on distance. Up to $0.08 \mathrm{~s}$, corresponding roughly to $100 \mathrm{~km}, \kappa$ is systematically lower for the vertical component, whereas at greater distances it is similar in both components. As a result, at $R=0$ the ratio of $\kappa_{0}^{h} / \kappa_{0}^{v}$ is around 1 at depth and 1.4 at the surface. This effect may be explained by observing Figure 9a: for the vertical component (triangles), the ratio of surface-to-downhole $\kappa$ does not depend on distance (it is 


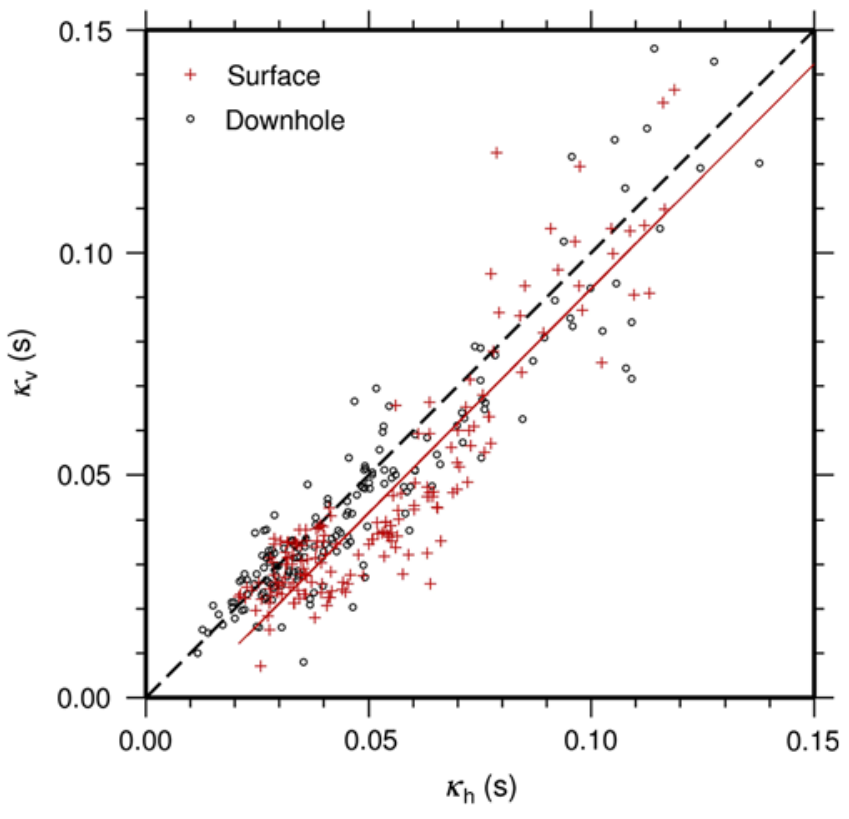

Figure 11. Comparison of individual $\kappa$ values for the vertical and horizontal component at the surface (dashes) and at depth (circles). The dashed line indicates a 1:1 relationship, which holds true at depth. The solid line indicates the tendency of the vertical $\kappa_{S}$ to be lower than the horizontal $\kappa_{S}$ at the surface. The color version of this figure is available only in the electronic edition.

scattered around 1 irrespective of distance), contrary to what was observed for the horizontal (circles). In this paper we do not propose full $\kappa$ models for the vertical component. This would require making an assumption as to the slope of the new model, for instance assuming similar $Q$ for $S$ waves in all directions and thus constraining the regression slope using data from all components.

\section{Alternatives to the Use of Distance}

We also searched for tools that may be used as alternatives to distance. Such tools are not proposed here as replacements for the usual procedure if the event locations are

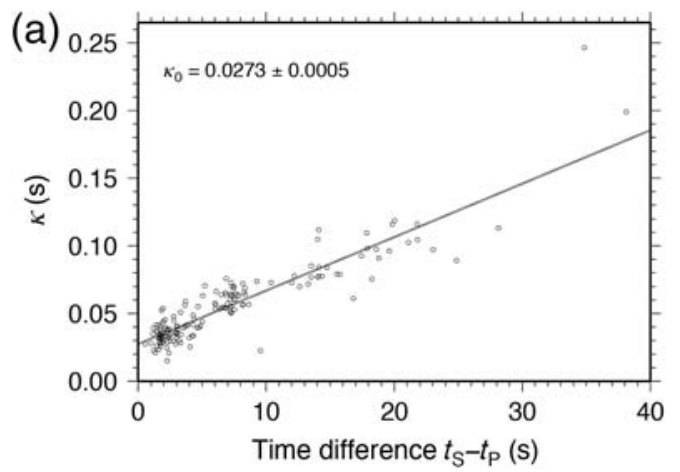

known but rather as relatively faster and easier methods to derive a first estimate of $\kappa_{0}$ at a given site.

\section{Time Difference between $S$ and $P$ Arrivals}

Having picked the $P$ and $S$ arrivals manually on each record, we used the difference in $S$ and $P$ arrival times as a measure of distance and recomputed the regressions to get $\kappa$ models with respect to $\left(t_{s}-t_{p}\right)$. The results are shown in Figure 12a. The linear fit $\left(R^{2}\right)$ is similar to that of Figure 5. We suggest that this alternative technique might be used for a first estimate of $\kappa_{0}$ directly from arrival times in cases where seismic catalogs and earthquake locations are not available or not dependable, or if there is no time to search catalogs or not enough stations to locate the events. It is also possible to get an estimate of $\kappa_{R}$. Assuming that the relation in $P$ - and $S$-wave velocities in the crust is $V_{P} / V_{S}=\sqrt{3}$, and that the path followed by the waves is a straight line, the source-to-site distance can be related to the times as $R=V_{S}\left(t_{s}-t_{p}\right) \sqrt{3} /(\sqrt{3}-1)$. Assuming an average crustal velocity of $3.5 \mathrm{~km} / \mathrm{s}$ in the area (Hatzidimitriou, 1993; Papazachos and Nolet, 1997), we can arrive at a $\kappa$ model as a function of "equivalent distance" with $\kappa_{R}=0.0048$ (Fig. 12b).

\section{Events at Very Short Distances}

Rebollar (1990) studied data from two hard rock sites in Mexico. For hypocentral distances up to $30 \mathrm{~km}$ he found that $\kappa$ showed no increase with distance, so he derived only $\kappa_{0}$ values (assuming zero slope). Singh et al. (1982) also considered that for epicentral distances between 8 and $50 \mathrm{~km} t^{*}$ was virtually independent of distance. Thus, in this section we investigate whether the use of records coming exclusively from nearby earthquakes might serve as a tool for an initial estimate of $\kappa_{0}$, the idea being that they could provide an average without the need of regression. This approach might have the advantage that in a site similar to Aegion in terms of seismicity, one would not need to wait long to record a fair number of small nearby events; it is

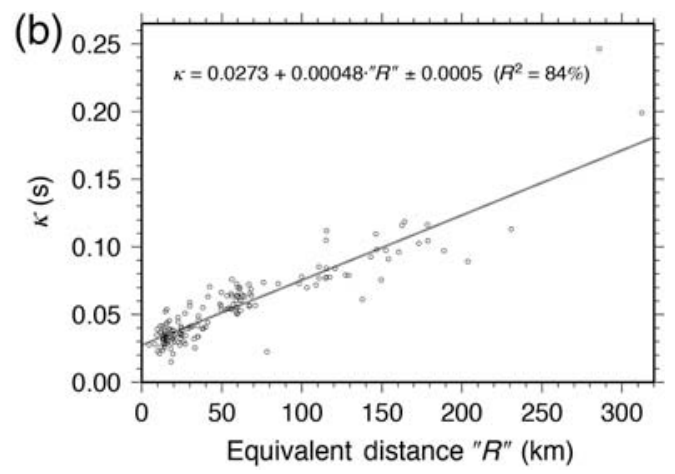

Figure 12. Distribution of individual $\kappa$ values with (a) $S-P$ arrival time difference and (b) equivalent distance based on the velocity model, as computed for the surface station. Regressed lines (average \pm 1 standard deviation) resulting from robust individual regressions are plotted onto the data points. Formulas show average line equations, standard deviation, and coefficient of correlation. 
the distant large events needed to define the slope that would occur less frequently.

We tested this idea on our data set. Looking at Figure 4b, we see that the linear increase of $\kappa$ with $R_{h}$ is not clear for distances below $30 \mathrm{~km}$ (similar to what Rebollar, 1990, noted), especially given the scatter. If $\kappa$ is indeed constant for these distances, then we can apply the averaging approach and avoid the regression. This approach leads to average values of $\kappa_{0}$ equal to 0.035 and $0.030 \mathrm{~s}$ for the surface and downhole station, respectively, which are 5 and $7 \mathrm{~ms}$ higher than the regression results. To explain this discrepancy we perform a nonparametric regression, where no assumptions are made as to the kind of dependence of $\kappa$ values with distance. We find that, despite the visible scatter in the data at short distances (which may be partly due to the location errors causing increased uncertainty in short distance estimates), $\kappa$ does indeed increase with distance even at short distances. The nonparametric regression yields $\kappa_{0}$ similar to the regression of equation (5). This means that the form of equation (5) did not force a distance dependence onto the data. Thus, for this data set, in contrast to the data sets mentioned in the beginning of this section, the averaging approach slightly overestimates the results of the AndersonHough approach. The alternative idea of using only nearby events to determine $\kappa$ may be more in line with the notion that it is these events that mostly control the seismic hazard in an area. Indeed, some recent alternative methods of computing $\kappa$ consider distances $<20-50 \mathrm{~km}$ (L. Al Atik and N. Abrahamson, personal comm., 2012). This work, however, focuses on how to implement the original definition of $\kappa$ given by Anderson and Hough (1984) and not on how to choose among the different definitions that have been proposed since. We only add that the variability discussed herein and stemming from the differences in implementing the original definition can only increase if one also considers the various alternative definitions as well.

\section{Result Variability, Comparisons, and Recommendations}

Variability due to Computation Process: Implications for GMPEs

Figure 13 gives a visual comparison of the variability of $\kappa$ in the computations we have presented herein (for all computed regression parameters in detail, see (E) Table $\mathrm{S} 1$ of the electronic supplement to this article). In Figure 13a, we plot all the $\kappa_{0}$ values at the surface and downhole station according to the different hypotheses and methods implemented. In Figure 13b, we do the same for the slope, $\kappa_{R}$, and in Figure $13 \mathrm{c}$ for the standard deviation. In the graphs the color code corresponds to the four combinations according to whether epicentral or hypocentral distance is used and whether the regression is standard linear or weighted robust. The cases shown on the horizontal axis correspond to the following cases examined:
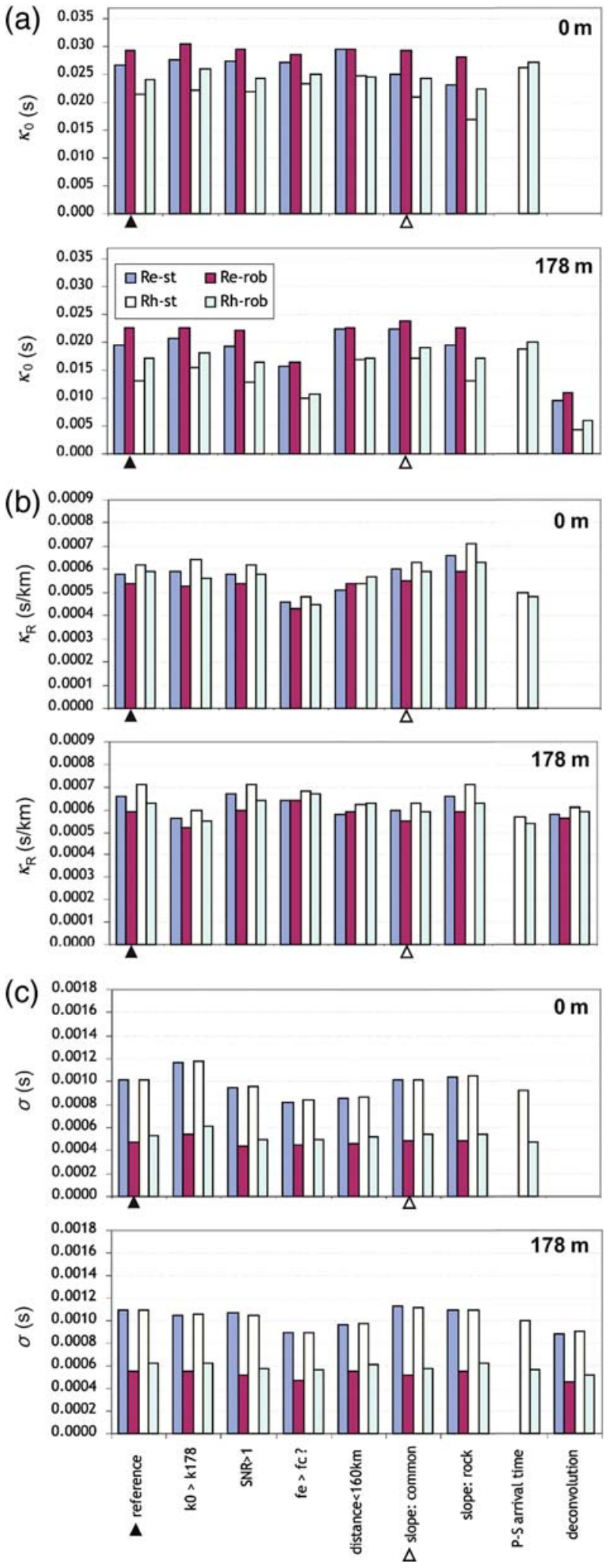

Figure 13. Variability of computed results for the surface and downhole station according to the different assumptions made during the computation process: (a) $\kappa_{0}$ values, (b) $\kappa_{R}$ values, and (c) standard deviation values. Preferred values are marked with an arrow (black if only surface records are available, white for simultaneous surface and downhole records). The color version of this figure is available only in the electronic edition. 
- the initial data set used here as reference (180 crustal events, each station studied independently);

- the data set consisting of the 128 events for which the criterion $\kappa_{0 \mathrm{~m}}>\kappa_{178 \mathrm{~m}}$ holds true;

- a different SNR criterion: 1 instead of 3;

- without ad hoc check for the corner frequency;

- exclusion of data at long distances (over $160 \mathrm{~km}$ );

- the two alternative cases where the slope was constrained: multiple regression with data from all stations and individual regressions with the slope fixed to the rock value;

- the use of $S-P$ times to estimate distance;

- the deconvolution based on the theoretical transfer function (results are only compared to rock).

Focusing on the variability of our estimates, $\kappa_{0}$ values may vary greatly depending on the combination of assumptions made during the computation. The type of regression causes $\kappa_{0}$ results to vary up to $30 \%$. This variation is significant given the uncertainty in the results: in these graphs error bars of \pm 1 standard deviation are around 5\%-10\% depending on the type of regression and station (for the robust regression the scatter is about half that of the standard regression, as seen in Fig. 13c). The largest variation is due to the type of distance. The use of hypocentral distance yields systematically lower $\kappa_{0}$ in all cases, though more so at rock level. This variation is $30 \%$ on average but may be up to $50 \%$ and is significant because it is comparable to the difference in $\kappa_{0}$ values between the surface and downhole rock station, which is on average about $40 \%$ (ranging from 20\% to $70 \%$ ), that is, comparable to the site effect itself. This site effect is comparable to what was found in other studies that compared downhole to surface $\kappa$ estimates: Oth et al. (2011) and Van Houtte et al. (2011) both found a difference of about $50 \%$ on average between surface records and downhole rock at depths of 100 or $200 \mathrm{~m}$. In any case, the fact that even at rock level $(178 \mathrm{~m})$ we still find a considerable $\kappa_{0}$ value is an indication of the deeper origin of $\kappa$. Indeed, both the seminal paper of Anderson and Hough (1984) as well as much more recent works (Campbell, 2009; Van Houtte et al., 2011) suggest that $\kappa_{0}$ may be affected by underlying formations not only down to a few hundreds of meters but as deep as a few kilometers.

In the introduction we mentioned some of the applications for which $\kappa$ (and most often, $\kappa_{0}$ ) is used as an input parameter. In the main body of this work we have shown the variability of values that $\kappa_{0}$ can assume according to the assumptions made during the computation with the AndersonHough method, even for a single site, and for a relatively large data set that is rather complete as to distances and with a good linear fit. Given that in this single study there is such variability and that there is no agreed procedure for the computation of $\kappa$, it is reasonable to believe that the results of published studies to date may depend on such assumptions and, if so, are not necessarily uniform across literature. For applications that use $\kappa$ as an input parameter, such as GMPEs, this could affect the precision and variability of their results. If the uncertainty around the mean value of $\kappa$ is associated to the particular technique used to compute it, then this technique affects the uncertainty of the overall result (here we call "technique" the set of choices made in implementing the Anderson-Hough method; in a broader sense it can also be any of the alternative approaches defined since that model). For instance, say a $\kappa_{0}$ estimate is required for rock conditions at Aegion. One researcher may choose to use epicentral distances, perform the regression with the robust weighing scheme, use a strict SNR value of 3, and accept only data for which $\kappa_{0 \mathrm{~m}}>\kappa_{178 \mathrm{~m}}$. Another may choose to use hypocentral distances, the standard regression method, an SNR of 1, and not check the $\kappa_{0 \mathrm{~m}} / \kappa_{178 \mathrm{~m}}$ ratio. Even if the two researchers use the same data set, choose the same parameters from the catalogs, and make the same pickings, their estimates would be $0.0228 \pm 0.0006 \mathrm{~s}$ and $0.0129 \pm 0.0011 \mathrm{~s}$, respectively. The difference in the mean values provided will be $0.0099 \mathrm{~s}$, and this "intratechnique" uncertainty is at least five times the error bar width of any of the individual estimates. Such values could constitute $\kappa_{0}$ values for host sites, that is, sites where data is readily available, and could then be used to calibrate GMPEs through the host-to-target method for target sites, where data are sparse, or for generic hard rock reference sites. The precision of the host-to-target adjustment process could benefit from a decrease in the variability of the $\kappa$ estimates at the host sites. We believe that a better understanding of the factors contributing to the variability could lead to a more conscious use of the results in applications such as these. Thus, in future, when using $\kappa$ estimates in applications such as ground-motion prediction, we believe it may be worthwhile to ask the question: how were they computed?

\section{Comparisons with Literature}

We will compare our results with certain empirical correlations that have been developed for $\kappa_{0}$ with respect to $V_{S 30}$. For the downhole rock (assuming it is equivalent to rock outcrop with $V_{S 30}=1000 \mathrm{~m} / \mathrm{s}$ ), the following predictions can be made for the median value: $\kappa_{0}=0.026 \mathrm{~s}$ according to the global database of Chandler et al. (2006), $0.022 \mathrm{~s}$ according to Silva et al. (1998), who worked with California data, $0.021 \mathrm{~s}$ according to Van Houtte et al. (2011), 0.017 or $0.020 \mathrm{~s}$ according to the two correlations of Edwards et al. (2011), based on Swiss data, and $0.018 \mathrm{~s}$ according to Drouet et al. (2010), who worked with French data. Taking into account their variation, the predictions are in reasonable agreement with our downhole results. For the surface station, on the other hand, the low $V_{S 30}$ value $(200 \mathrm{~m} / \mathrm{s})$ is out of range of the data used to create the correlations. Van Houtte et al. (2011) also propose correlations for $V_{S 5}, V_{S 10}, V_{S 20}, V_{S \text { mean }}$, and $f_{o}$, which overestimate $\kappa_{0}$ at the surface of CORSSA, possibly due to the data range being pushed to its lower limits. Here we note that existing empirical correlations exhibit a large scatter (most have a standard deviation of around $\pm 0.012 \mathrm{~s}$ for $V_{S 30}=1000 \mathrm{~m} / \mathrm{s}$ ). 
This scatter may be partly due to the computation-driven variability in $\kappa$ estimates that this paper investigates.

We also compare $\kappa$ computed at CORSSA with values found in the literature for regions near the Gulf of Corinth. Margaris and Boore (1998) found individual $\kappa$ (not $\kappa_{0}$ ) values for soils ranging from 0.04 to $0.06 \mathrm{~s}$ for surface records at distances of $23-33 \mathrm{~km}$. In that distance range our values are comparable. Margaris and Hatzidimitriou (2002) found $\kappa$ values in Greece of $0.065 \mathrm{~s}$ for soft soils and $0.035 \mathrm{~s}$ for generic rock. Hatzidimitriou et al. (1993) found $\kappa_{0}$ of $0.06 \mathrm{~s}$ in Greece. Dimitriu et al. (2001) computed $\kappa_{0}$ higher than $0.04 \mathrm{~s}$ for soils at Lefkas, which is higher than our surface estimates, but according to the authors their results may have been affected by nonlinearity due to high PGAs. Finally, Tselentis (1993) studied $\kappa$ ad hoc at the Western CorinthPatras rift using records on limestone outcrop and making regressions with hypocentral distance. He computed $\kappa_{0}$ of $0.04 \pm 0.02 \mathrm{~s}$ and $\kappa_{R}$ of around $0.0012 \mathrm{~s} / \mathrm{km}$. He considers these values characteristic of rock. Our study yields significantly lower $\kappa$ though we expected the opposite, given that our downhole rock station is in conglomerate (180-m depth, $V_{S}=1000 \mathrm{~m} / \mathrm{s}$ ), which in Aegion lies well above the limestone layer, which is considered as seismic bedrock (700-m depth; Apostolidis et al., 2006). This significant difference even for the rock estimates leads us to consider the variation of $\kappa_{0}$ for rock sites, which is of particular interest nowadays in the context of empirical GMPEs and host-to-target adjustment methods between rock and hard rock sites. Rebollar et al. (1991) compared $\kappa_{0}$ values for rock sites found across literature and concluded that their large variability is due not only to rock type but also to rock hardness; Tselentis (1993) reiterated this point, whereas Fernández et al. (2010) added the degree of fracturing and erosion as another factor. The results mentioned by Tselentis (1993) (Anderson and Hough, 1984; Rebollar, 1990; Rebollar et al., 1991), are reproduced in Figure 14; to them we add recent results from the same basic method of Anderson and Hough (1984): Margaris and Hatzidimitriou (2002), Douglas et al. (2010), Van Houtte et al. (2011), and the present study. Finally, we add the $\kappa$ values estimated by Oth et al. (2011); although they perform an inversion rather than use the traditional approach, they have made one of the only two studies of $\kappa$ on downhole data that we know of. As seen from the Figure 14, the variation in $\kappa_{0}$ estimates for surface rock is overall large. We be-

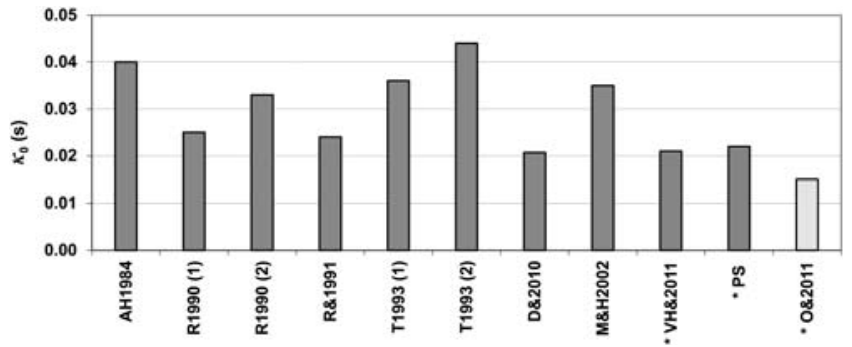

Figure 14. Variability of average $\kappa_{0}$ values estimated for rock sites by different researchers using the traditional Anderson-Hough method: Anderson and Hough (1984), Rebollar (1990), Rebollar et al. (1991), Tselentis (1993), Margaris and Hatzidimitriou (2002), Douglas et al. (2010), Oth et al. (2011), Van Houtte et al. (2011), and the present study. Downhole sites, asterisks.

lieve this variability might be due not only to the rock type, hardness, and erosion but also possibly to the methodology used in the computation process of $\kappa$, as shown through the factors examined in the present study. (Table 4 shows a few of the parameters considered differently in these studies.)

There is also the issue of whole-path attenuation and how that is related to $\kappa$. In this study we have not attempted to correct for whole-path attenuation. Most studies that follow the Anderson and Hough (1984) approach to compute $\kappa$ on accelerograms in log-linear space do not correct accelerograms for $Q$ before estimating $\kappa$, which means that $Q$ is partly present in the results (Douglas et al., 2010). It is possible to compute a "pure" site-only $\kappa$ if the correction is made beforehand, as did Edwards et al. (2011) and Margaris and Boore (1998); the latter found that this correction had a very small effect on the final $\kappa_{0}$ values. If the correction is not made then it is possible to compute $Q$ a posteriori based on the $\kappa$ results (e.g., Hough et al., 1988). In our case, based on the slope of the regressed lines and the crustal $V_{S}$ model, we can infer a frequency-independent $S$-wave $Q$ of 500 for the range of frequencies studied here. Our results regarding regional attenuation are in good agreement with independent studies. For the back-arc area of the Hellenic Arc (which includes the Gulf of Corinth), Polatidis et al. (2003) found $Q$ to be frequency dependent with an average value of 553 for bands centered around $12 \mathrm{~Hz}$. Hatzidimitriou et al. (1993) found a frequency-independent $Q$ of 600 for the granitic bedrock structure in Greece (at depths $>2-3 \mathrm{~km}$ ). We think that

Table 4

Different Parameters Considered in Studies Computing $\kappa$ on Rock Outcrop

\begin{tabular}{lccrrll}
\hline \multicolumn{1}{c}{ Reference } & $\mathrm{R}$ type & $R_{\max }(\mathrm{km})$ & $\mathrm{SNR}$ & \multicolumn{1}{c}{$N_{\text {rec }}$} & \multicolumn{1}{c}{ Location } & \multicolumn{1}{c}{ Rock Type } \\
\hline Anderson and Hough (1984) & $R_{e}$ & 180 & & 100 & California & Igneous/metamorphic \\
Rebollar (1990) & $R_{h}$ & 30 & & 35 & Baja California & Granite \\
Rebollar et al. (1991) & $R_{h}$ & 90 & & 100 & Oaxaca & Crystalline \\
Tselentis (1993) & $R_{h}$ & 65 & 5 & 45 & Corinth-Patras & Limestone \\
Douglas et al. (2010) & $R_{e}$ & 350 & $3 /$ visual & 250 & France & Various \\
Van Houtte et al. (2011) & $R_{h}$ & 100 & 1 & 4554 & Japan & Various (downhole) \\
Margaris and Hatzidimitriou (2002) & $R_{h}$ & 115 & & 25 & Greece & $V_{S 30}>750 \mathrm{~m} / \mathrm{s}$ \\
\hline
\end{tabular}




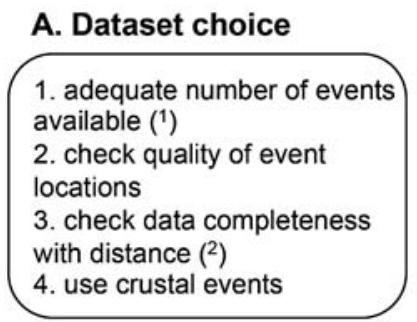

\section{B. Record processing}

1. visual quality inspection
2. adequate noise window
3. pick $P, S$
4. realistic S-window choice
(minimum duration $3 \mathrm{~s}$ )

\section{Spectrum processing}

1. compute $f_{c}$ or pick on displacement

2. pick $f_{e}, f_{x}$ on acceleration

3. check: $f_{e}>f_{c}$

4. check: $f_{x}<f_{\text {(flat instr. response) }}$

5. check: SNR $>3$

6. check: $\Delta \mathrm{f}>10 \mathrm{~Hz}$

7. $\kappa=-\lambda / \pi$

8. average 2 horizontals if similar ( $<20 \%$ difference)

\section{D. $\kappa$ model regression}

1. use epicentral distance 2. check linear form visually

3. esp. if there is only one analyst, or if dataset is small ( ${ }^{1}$ e.g. $N<30$ ) or poor at large distances $\left({ }^{2}\right)$ : consider weighed robust regression 4. check linear form e.g. through $R^{2}>65 \%$

5. check slope with independent $Q$ estimates 6 . if there is data redundancy (downhole/nearby stations): make a multiregression to achieve the same slope for all $\kappa$ models

7. do not correct with theoretical transfer function (unless there is significant high-frequency resonance) but avoid first resonant peaks

\section{E. Special cases}

1. for 1D sensor: vertical component approximation, esp. for rock

2. if event locations unavailable: P-S arrival approximation

Figure 15. Suggested flowchart for the computation of $\kappa$ following the Anderson and Hough (1984) definition.

making such comparisons with independent $Q$ estimates may help verify the accuracy of the slope computed with the Anderson and Hough (1984) method. In our case the linear fit of the data was good. In cases where this is not as clear, knowledge of $Q$ at high frequencies could help constrain the results of the regression.

\section{Recommended Procedure for $\kappa$ Computation}

Given the variability of our results depending on the computation procedure, we believe it may be helpful to try to standardize this procedure to the extent possible. In the flowchart of Figure 15 we provide some recommendations based on our findings. Among the approaches mentioned in the preceding analyses, we chose those that we think yield more robust estimates (use epicentral distance, avoid paths passing through the mantle, use weighted regression, SNR $>3$, explicit check to avoid corner frequency and source effects, and apply constraints for common regional $Q$ effects). It would be useful to verify them through computations made on other data sets as well. According to these recommendations the preferred results we get for CORSSA are:

- $\kappa_{0}=0.0294 \pm 0.0005 \mathrm{~s}$ (surface) and $\kappa_{0}=0.0226 \pm$ $0.0005 \mathrm{~s}$ (rock) viewing stations independently, that is to say, if we only had information on one station;

- $\kappa_{0}=0.0293 \pm 0.0005 \mathrm{~s}$ (surface) and $\kappa_{0}=0.0239 \pm$ $0.0005 \mathrm{~s}$ (rock) for the common slope constraint proposed for the case of an array.

The suggested methods are marked in the histograms of Figure 13 with arrows: black for the basic case of independent stations and white for the special case where downhole records are also available and thus the slope is constrained. We should mention here that the values of standard deviation that accompany these results $(0.0005 \mathrm{~s})$ are relatively low thanks to the use of the robust method of regression, which downweighs the outliers, and also due to the volume of data used. The standard deviation of the mean for an alternative method will depend on that particular method. Finally, even for data sets that do not fit the linear model well and do not satisfy steps D2 and D4 of the flowchart (Fig. 15), our recommendations can still be used as a guide, as long as one substitutes equation (5) with a more appropriate data-derived function of $\kappa$ with epicentral distance and applies it to all stations concerned as per step D6.

\section{Conclusions}

We estimate $\kappa$ for 180 earthquakes at a vertical array where conditions vary from soft deposits to hard conglomerate. We study the variability of $\kappa$ depending on the assumptions made in the computation process after Anderson and Hough (1984), including the definition of distance, the type of linear regression with distance, the choice of the data set, the SNR ratio, the choice of frequency range, the removal of site effects, and the use of constraints with regard to the regional dependence.

We suggest the use of epicentral distance for the extrapolation to $\kappa_{0}$. We find that the choice of distance systematically affects $\kappa_{0} ; \kappa_{0}$ is significantly lower (up to $20 \%$ at the surface and up to $40 \%$ at rock) if hypocentral rather than epicentral distance is used. This difference is larger than the standard deviation of the estimates. The linear formulation including site and distance $\left(\kappa=\kappa_{0}+\kappa_{R} R\right)$ is a good approximation for our data $\left(R^{2} \sim 80 \%\right)$. To overcome the possible subjectivity in the manual picking of the records, we suggest the use of a robust weighed regression scheme. This option also permits the use of data at large distances, where the data set is incomplete and $Q$ variations can increase scatter, without introducing a bias.

We find no correlation of $\kappa$ with azimuth. On the contrary, we find it is possible for event clusters to exhibit large scatter in $\kappa$ values and for events coming from very different azimuths and different source-to-site geometries to exhibit similar kappas. We find no correlation between $\kappa$ and magnitude. Subduction seismicity yields much larger scatter in $\kappa$ and higher $Q$. There are also other implicit procedures that affect $\kappa$ computation. We find that if the SNR criterion is not applied strictly (i.e., if $f_{x}$ is too high), it may lead to underestimation of $\kappa_{0}$ due to the inclusion of noise in the spectral window. On the other hand, there is also the danger of picking an $f_{e}$ that is too low. This may happen if the picking is 
based solely on the observation of acceleration spectra without taking into account the corner frequency.

We attempt to correct surface records for site resonance by deconvolving them to rock level using the site's theoretical transfer function. This is not a straightforward process, and even for a site as well documented as CORSSA it may introduce further uncertainty. Here, surface station spectra projected to depth by deconvolving the geotechnical structure underestimate $\kappa_{0}$ observed at the rock sensor by as much as $50 \%$. This we attribute to possible artifacts in the theoretical computation of site response or lack of knowledge of the soil profile at very high frequencies, such as those used here (up to $60 \mathrm{~Hz}$ ). At this deep site, the correction is probably only helpful at much lower frequencies.

We also study two alternative ways for a quick initial estimation of $\kappa$ : the difference between $S$ and $P$ arrival times as a substitute for distance and the average of individual $\kappa$ values at very short distances. The former gives a good approximation of $\kappa_{0}$ and the latter yields an upper bound for $\kappa_{0}$. For the vertical component we found that at depth (rock conditions) $\kappa_{0}$ is similar in all components, though at the surface (soil) it is around $40 \%$ higher in the horizontal.

Finally, a new kind of uncertainty stems from the fact that we have several stations at the same location and from the constraint this may impose on regional dependence. Computing $\kappa$ models for each station independently we find that, though $\kappa_{0}$ values correlate to $V_{S}$ as expected, $\kappa_{R}$ is slightly different between rock and soil. Because the slope is assumed to be a regional effect, we constrain it to a common value for all stations. We use three approaches: first, a common slope is assumed for all array stations; then, we fix the slope to the rock value; and finally, after observing that the difference in slopes is due to the dependence of $\kappa_{\text {soil }} / \kappa_{\text {rock }}$ with distance, we recompute $\kappa$ using only records for which $\kappa_{\text {soil }} / \kappa_{\text {rock }}>1$. Thus, we conclude that data availability (e.g., here, the number of stations used) becomes yet another factor affecting the final $\kappa$ estimate.

Overall, in this sensitivity study we find that the final estimate depends on the assumptions made during the computation process, one of the most important being the distance definition. The variability of $\kappa_{0}$ estimates according to such assumptions is significant and larger than the error bars accompanying each estimate. Because there is no agreed procedure for $\kappa_{0}$ estimation and assumptions vary across literature, we believe $\kappa$ estimates published up to now and used as input in applications such as GMPE adjustments, $\kappa-V_{S 30}$ correlations, or stochastic simulations need to be evaluated on a case-by-case basis before being blindly used in such applications. Regarding computation of $\kappa$ in the future, we make some recommendations as to how this might be achieved in a more controlled and homogeneous way when implementing the traditional Anderson-Hough method. If, however, one also takes into account the more recent definitions of $\kappa$ proposed as alternatives to the traditional method (use of response spectra, full broadband inversions, use of displacement spectra for small events, etc.), the variability of $\kappa$ values in the literature is probably even larger.

\section{Data and Resources}

The accelerograms used in this study were produced by the CORSSA array, which operated in the framework of an agreement between Aristotle University of Thessaloniki, Greece, National Kapodistrian University of Athens, Greece, and Institut de Radioprotection et de Sûreté Nucléaire, France. Signal processing benefitted significantly from SAC2008 (Goldstein et al., 2003; http://www.iris.edu/software/sac, last accessed December 2012; Goldstein and Snoke (2005); SAC Availability for the IRIS Community, Incorporated Institutions for Seismology Data Management Center Electronic Newsletter; http://www.iris.edu/news/newsletter/vol7no1/ page1.htm, last accessed December 2012). Some plots were made using Generic Mapping Tools v. 3.4 (http://www.soest .hawaii.edu/gmt, last accessed December 2012; Wessel and Smith, 1998). The four main Greek earthquake catalogs used are the following: Aristotle University of Thessaloniki, http:// geophysics.geo.auth.gr/ss/CATALOGS/preliminary/finalcat .cat (last accessed December 2011); National Kapodistrian University of Athens, http://www.geophysics.geol.uoa.gr/ catalog/catgr_20002008.epi (last accessed December 2011); National Observatory of Athens, http://www.gein.noa.gr/ services/cat.html (last accessed: December 2011); and Patras Seismological Laboratory, http://seismo.geology.upatras.gr/ (last accessed December 2011). We have also made use of the New Global Crustal Model CRUST2.0 (http://igppweb .ucsd.edu/ gabi/rem.html; last accessed December 2012) as a reference for crustal thickness.

\section{Acknowledgments}

The installation of CORSSA was funded by European research project CORSEIS (EVG1-1999-00002) and its maintenance continued through the CORSSA agreement. The research for this paper was conducted under the funding of French national research project ANR DEBATE (Risk'nat 2008: ANR-08-RISKNAT-01) during a postdoctoral stay of O. J. K. at the Seismic Risk and Facility Safety Assessment Unit (BERSSIN) of IRSN, France. The drafting of the paper was finalized under the funding of European research project SHARE (Seismic Hazard Harmonization in Europe, Contract Number 226967) during a postdoctoral stay at Joseph Fourier University-CNRS, Grenoble, France. We are thankful for discussions with BERSSIN researchers, especially M. Lancieri and O. Scotti. We have also benefitted from discussions with A. Kiratzi, P.-Y. Bard, N. Abrahamson, and R. Archuleta. We thank J. Anderson for his clarifications on the use of epicentral distance. We thank S. Arnaouti and G. Ameri for valuable help with regression algorithms and F. Cotton for a thoughtful review of the manuscript and many discussions. An anonymous reviewer provided useful remarks and corrections that helped improve and clarify the text. Finally, warm thanks go to Glenn Biasi for his meticulous and insightful reviews, comments, and constructive criticisms; this work is the better for them.

\section{References}

Anderson, J. G. (1991). A preliminary descriptive model for the distancedependence of the spectral decay parameter in southern California, Bull. Seismol. Soc. Am. 81, 2186-2193. 
Anderson, J. G., and S. E. Hough (1984). A model for the shape of the Fourier amplitude spectrum of acceleration at high frequencies, Bull. Seismol. Soc. Am. 74,1969-1993.

Anderson, J. G., and J. R. Humphrey (1991). A least squares method for objective determination of earthquake source parameters, Seismol. Res. Lett. 62, 201-209.

Apostolidis, P., D. Raptakis, K. Pandi, M. Manakou, and K. Pitilakis (2006). Definition of subsoil structure and preliminary ground response in Aigion city (Greece) using microtremor and earthquakes, Soil Dynam. Earthq. Eng. 26, 49-67.

Atkinson, G. M., and D. M. Boore (2006). Earthquake ground-motion prediction equations for Eastern North America, Bull. Seismol. Soc. Am. 96, 2181-2205.

Bassin, C., G. Laske, and G. Masters (2000). The current limits of resolution for surface wave tomography in North America, Eos Trans. AGU 81, F897.

Biasi, G. P., and K. D. Smith (2001). Site effects for seismic monitoring stations in the vicinity of Yucca Mountain, Nevada, MOL20011204 .0045 , a report prepared for the US DOE/University and Community College System of Nevada (UCCSN) Cooperative Agreement.

Boore, D. M. (1983). Stochastic simulation of high-frequency ground motions based on seismological models of the radiated spectra, Bull. Seismol. Soc. Am. 73, 1865-1894.

Boore, D. M. (2003). Simulation of ground motion using the stochastic method, Pure Appl. Geophys. 160, 635-676.

Boore, D. M., and W. B. Joyner (1997). Site Amplifications for Generic Rock Sites, Bull. Seismol. Soc. Am. 87, 327-341.

Brune, J. (1970). Tectonic stress and the spectra of seismic shear waves, J. Geophys. Res. 75, 4997-5009.

Campbell, K. W. (2003). Prediction of strong ground motion using the hybrid empirical method and its use in the development of ground motion (attenuation) relations in eastern North America, Bull. Seismol. Soc. Am. 93, 1012-1033.

Campbell, K. W. (2009). Estimates of shear-wave $Q$ and $\kappa_{0}$ for unconsolidated and semiconsolidated sediments in Eastern North America, Bull. Seismol. Soc. Am. 99, 2365-2392.

Castro, R. R., L. Trojani, G. Monachesi, M. Mucciarelli, and M. Cattaneo (2000). The spectral decay parameter $\kappa$ in the region of UmbriaMarche, Italy, J. Geophys. Res. 105, 23,811-23,823.

Chandler, A. M., N. T. K. Lamb, and H. H. Tsang (2006). Near-surface attenuation modelling based on rock shear-wave velocity profile, Soil Dynam. Earthq. Eng. 26, 1004-1014.

Cormier, V. F. (1982). The effect of attenuation on seismic body waves, Bull. Seismol. Soc. Am. 72, S169-S200.

Cotton, F., F. Scherbaum, J. J. Bommer, and H. Bungum (2006). Criteria for selecting and adjusting ground-motion models for specific target regions: Application to Central Europe and rock sites, J. Seismol. 10, 137-156.

Dainty, A. M. (1981). A scattering model to explain seismic $Q$ observations in the lithosphere between 1 and $30 \mathrm{~Hz}$, Geophys. Res. Lett. 8, 1126-1128.

Dimitriu, P., N. Theodulidis, P. Hatzidimitriou, and A. Anastasiadis (2001). Sediment non-linearity and attenuation of seismic waves: a study of accelerograms from Lefkas, western Greece, Soil Dynam. Earthq. Eng. 21, 63-73.

Douglas, J., H. Bungum, and F. Scherbaum (2006). Ground motion prediction equations for southern Spain and southern Norway obtained using the composite model perspective, J. Earthq. Eng. 10, 33-72.

Douglas, J., P. Gehl, L. F. Bonilla, and C. Gélis (2010). A $\kappa$ model for mainland France, Pure Appl. Geophys. 167, 1303-1315.

Drouet, S., F. Cotton, and P. Gueguen (2010). $V_{S 30}, \kappa$, regional attenuation and $M_{\mathrm{w}}$ from accelerograms: Application to magnitude 3-5 French earthquakes, Geophys. J. Int. 182, 880-898.

Edwards, B., D. Fah, and D. Giardini (2011). Attenuation of seismic shear wave energy in Switzerland. Geophys. J. Int. 185, 967-984.

Fernández, A. I., R. R. Castro, and C. I. Huerta (2010). The spectral decay parameter kappa in Northeastern Sonora, Mexico, Bull. Seismol. Soc. Am. 100, 196-206.
Frankel, A., and L. Wennerberg (1987). Energy-flux model of seismic coda: Separation of scattering and intrinsic attenuation, Bull. Seismol. Soc. Am. 77, 1223-1251.

Futterman, W. I. (1962). Dispersive body waves, J. Geophys. Res. 67, 5279-5291.

Gentili, S., and G. Franceschina (2011). High frequency attenuation of shear waves in the southeastern Alps and northern Dinarides, Geophys. $J$. Int. 185, 1393-1416.

Goldstein, P., D. Dodge, M. Firpo, and L. Minner (2003). SAC2000: Signal processing and analysis tools for seismologists and engineers, Invited contribution to The IASPEI International Handbook of Earthquake and Engineering Seismology, W. H. K. Lee, H. Kanamori, P. C. Jennings, and C. Kisslinger (Editors), Academic Press, London.

Halldorsson, B., and A. S. Papageorgiou (2005). Calibration of the specific barrier model to earthquakes of different tectonic regions, Bull. Seismol. Soc. Am. 95, 1276-1300.

Hanks, T. C. (1982). $f_{\max }$, Bull. Seismol. Soc. Am. 72, 1867-1879.

Hatzidimitriou, P. M. (1993). Attenuation of coda waves in Northern Greece, Pure Appl. Geophys. 140, 63-78.

Hatzidimitriou, P., C. Papazachos, A. Kiratzi, and N. Theodulidis (1993). Estimation of attenuation structure and local earthquake magnitude based on accleration records in Greece, Tectonophysics 217, 243-253.

Hough, S. E., and J. G. Anderson (1988). High-frequency spectra observed at Azna, California: Implications for $Q$ structure, Bull. Seismol. Soc. Am. 78, 692-707.

Hough, S. E., J. G. Anderson, J. Brune, F. Vernon, J. Berger, J. Fletcher, L. Haar, T. Hanks, and L. Baker (1988). Attenuation near Anza, California, Bull. Seismol. Soc. Am. 78, 672-691.

Joyner, W. B., R. E. Warrick, and T. E. Fumal (1981). The effect of Quaternary alluvium on strong ground motion in the Coyote Lake, California, earthquake of 1979, Bull. Seismol. Soc. Am. 71, 1333-1349.

Kennet, B. L. N. (1983). Seismic Wave Propagation in Stratified Media. Cambridge University Press, New York, 242 pp.

Kilb, D., G. Biasi, J. G. Anderson, J. Brune, Z. Peng, and F. L. Vernon (2012). A comparison of spectral parameter kappa from small and moderate earthquakes using Southern California ANZA seismic network data, Bull. Seismol. Soc. Am. 102, 284-300.

Konno, K., and T. Ohmachi (1998). Ground-motion characteristics estimated from spectral ratio between horizontal and vertical components of microtremor, Bull. Seismol. Soc. Am. 88, 228-241.

Ktenidou, O.-J. (2010). Theoretical and instrumental study of site and topographic effects on strong ground motion in Aegion, Ph.D. Thesis, Aristotle University Thessaloniki, Greece. available online at: http:// invenio.lib.auth.gr/record/124050/files/GRI-2010-5489.pdf (last accessed July 2012).

Ktenidou, O.-J., F. J. Chávez-García, and K. Pitilakis (2011). Variance reduction and signal-to-noise ratio: Reducing uncertainty in spectral ratios, Bull. Seismol. Soc. Am. 101, 619-634.

Lancieri, M., R. Madariaga, and F. Bonilla (2012). Spectral scaling of the aftershocks of the Tocopilla 2007 earthquake in Northern Chile, Geophys. J. Int., 189, 1, 469-480, doi: 10.1111/j.1365-246X.2011.05327.x.

Lay, T., and T. C. Wallace (1995). Modern Global Seismology, Academic Press, San Diego, California, 521.

Margaris, B. N., and D. M. Boore (1998). Determination of $\Delta \sigma$ and $\kappa_{0}$ from response spectra of large earthquakes in Greece, Bull. Seismol. Soc. Am. 88, 170-182.

Margaris, B. N., and P. M. Hatzidimitriou (2002). Source spectral scaling and stress release estimates using strong-motion records in Greece, Bull. Seismol. Soc. Am. 92, 1040-1059.

Mena, B., P. M. Mai, K. B. Olsen, M. D. Purvance, and J. N. Brune (2010). Hybrid broadband ground-motion simulation using scattering Green's functions: Application to large-magnitude events, Bull. Seismol. Soc. Am. 100, 2143-2162.

Oth, A., D. Bindi, S. Parolai, and D. Di Giacomo (2010). Earthquake scaling characteristics and the scale-(in)dependence of seismic energy-tomoment ratio: Insights from KiK-net data in Japan, Geophys. Res. Lett. 37, L19304, doi 10.1029/2010GL044572. 
Oth, A., D. Bindi, S. Parolai, and D. Di Giacomo (2011). Spectral analysis of K-NET and KiK-net data in Japan, part II: On attenuation characteristics, source spectra, and site response of borehole and surface stations, Bull. Seismol. Soc. Am. 101, 667-687.

Papageorgiou, A. S., and K. Aki (1983). A specific barrier model for the quantitative description of inhomogeneous faulting and the prediction of strong ground motion, part I: Description of the model, Bull. Seismol. Soc. Am. 73, 693-722.

Papazachos, C., and G. Nolet (1997). $P$ and $S$ deep velocity structure of the Hellenic area obtained by robust nonlinear inversion of travel times, $J$. Geophys. Res. 102, 8349-8367.

Parolai, S., and D. Bindi (2004). Influence of soil-layer properties on $k$ evaluation, Bull. Seismol. Soc. Am. 94, 349-356.

Pitilakis, K., K. Makropoulos, P. Bernard, F. Lemeiile, H. Lyon-Caen, C. Berge-Thierry, Th. Tika, M. Manakou, D. Diagourtas, D. Raptakis, P. Kallioglou, K. Makra, D. Pitilakis, and F. Bonilla (2004). The Corinth Gulf Soft Soil Array (CORSSA) to study site effects, C.R. Geoscience 336, 353-365.

Polatidis, A., A. Kiratzi, P. Hatzidimitrioua, and B. Margaris (2003). Attenuation of shear-waves in the back-arc region of the Hellenic arc for frequencies from 0.6 to $16 \mathrm{~Hz}$, Tectonophysics 367, 29-40.

Purvance, M. D., and J. G. Anderson (2003). A comprehensive study of the observed spectral decay in strong-motion accelerations recorded in Guerrero, Mexico, Bull. Seismol. Soc. Am. 93, 600-611.

Raptakis, D. G., M. V. Manakou, F. J. Chávez-García, K. A. Makra, and K. D. Pitilakis (2005). 3D configuration of Mygdonian basin and preliminary estimate of its site response, Soil Dynam. Earthq. Eng. 25, 871-887.

Rebollar, C. J. (1990). Estimates of shallow attenuation of the San Miguel fault, Baja California, Bull. Seismol. Soc. Am. 80, 43-46.

Rebollar, C. J., L. Munguía, A. Reyes, A. Uribe, and O. Jiménez (1991). Estimates of shallow attenuation and apparent stresses from aftershocks of the Oaxaca earthquake of 1978, Bull. Seismol. Soc. Am. 81, 99-108.

Silva, W., R. Darragh, N. Gregor, G. Martin, N. Abrahamson, and C. Kircher (1998). Reassessment of site coefficients and near-fault factors for building code provisions, Technical Report Program Element II: 98-HQGR-1010, Pacific Engineering and Analysis, El Cerrito.

Singh, S. K., R. J. Apsel, J. Fried, and J. N. Brune (1982). Spectral attenuation of $S H$ waves along the Imperial fault, Bull. Seismol. Soc. Am. 72, 2003-2016.
Singh, S. K., M. Ordaz, J. G. Anderson, M. Rodriguez, R. Quaas, E. Mena, M. Ottaviani, and D. Almora (1989). Analysis of near-source strongmotion recordings along the Mexican subduction zone, Bull. Seismol. Soc. Am. 79, 1697-1717.

Toro, G. R., N. A. Abrahamson, and J. F. Schneider (1997). Model of strong ground motions from earthquakes in central and eastern North America: Best estimates and uncertainties, Seismol. Res. Lett. 68, 41-57.

Tsai, C.-C. P., and K.-C. Chen (2000). A model for the high-cut process of strong motion accelerations in terms of distance, magnitude, and site condition: an example from the SMART 1 array, Lotung, Taiwan, Bull. Seismol. Soc. Am. 90, 1535-1542.

Tselentis, G.-A. (1993). Shallow attenuation in the West Corinth-Patras Rift, Greece, Bull. Seismol. Soc. Am. 83, 603-609.

Van Houtte, C., S. Drouet, and F. Cotton (2011). Analysis of the origins of $\kappa$ (Kappa) to compute hard rock to rock adjustment factors for GMPEs, Bull. Seismol. Soc. Am. 101, 2926-2941.

Wessel, P., and W. H. F. Smith (1998). New, improved version of the generic mapping tools released, EOS Trans. AGU 79, 579.

Bureau d'Evaluation des Risques Sismiques pour la Sûreté des Installations Nucléaires (BERSSIN)

Institut de Radioprotection et de Sûreté Nucléaire (IRSN)

BP 17, 92262 Fontenay-aux-Roses

Cedex, France

olga.ktenidou@obs.ujf-grenoble.fr

(O.-J.K., C.G.)

Université Paris Est

Institut Français des Sciences et Technologies des Transports

de l'Aménagement et des Réseaux (IFFSTAR)

Cité Descartes-14-20 bd Newton-77447

Champs-sur-Marne-Marne-la-Vallée

Cedex 2, France

(L.-F.B.) 